\title{
Symplectic model for piezoelectric wedges and its application in analysis of electroelastic singularities
}

\author{
J.-S. WANG† and Q.-H. QIN*+ \\ $\dagger$ Department of Mechanics, School of Mechanical Engineering, \\ Tianjin University, Tianjin 300072, China \\ tDepartment of Engineering, Australian National University, \\ Canberra, ACT 0200, Australia
}

(Received 10 April 2006; in final form 3 August 2006)

\begin{abstract}
In this paper, a symplectic model, based on the Hamiltonian system, is developed for analyzing singularities near the apex of a multi-dissimilar piezoelectric wedge under antiplane deformation. The derivation is based on a modified Hellinger-Reissner generalized variational principle or a differential equation approach. The study indicates that the order of singularity depends directly on the non-zero eigenvalue of the proposed Hamiltonian operator. Using the coordinate transformation technique and continuity conditions on the interface between two dissimilar materials, the orders of singularity for multi-dissimilar piezoelectric and piezoelectric-elastic composite wedges are determined. Numerical examples are considered to show potential applications and validity of the proposed method. It is found that the order of singularity also depends on the piezoelectric constant, in addition to the geometry and shear modulus.
\end{abstract}

\section{Introduction}

Piezoelectric materials are commonly used as sensors and actuators in adaptive structures owing to their electro-mechanical coupling effect [1]. In practical applications, piezoelectric elements are often bonded to other composite materials. In particular, they are often used in bimaterial structures or wedge structures [2-4], which may exhibit singular electro-mechanical fields on the interface of bimaterials or near the apex, induced by geometric and material discontinuities under strenuous mechanical and electrical loads [4-7]. The corresponding singular stresses and electric fields may even become infinite, leading to dielectric breakdown, debonding and fracture.

Singularity analysis of stress fields at the apex of elastic wedges has attracted great interest over the past decades [2-6]. In 1952, Williams [6] studied the stress singularities of an elastic wedge based on plane stress theory. Since then, several mathematical approaches have been presented to determine the order of stress singularity near the apex of elastic wedges, such as the eigenfunction expansion, Mellin transform and complex potential functions (see [7] for details).

*Corresponding author. Email: qinghua.qin@anu.edu.au 
For singularity analysis of piezoelectric wedges within the framework of Euclidean space, $\mathrm{Xu}$ and Rajapakse [8] studied the in-plane singular behaviour of electroelastic fields at the corner of piezoelectric wedges and junctions using extended Lekhnitskii's complex potential functions and Williams' eigenfunction expansion. Using a similar procedure, Chue and Chen [9] analyzed stress singularities of a piezoelectric composite wedge under generalized plane deformation. They subsequently extended their results to the antiplane problem of a piezoelectric bonded wedge using the Mellin transform [10] and eigenfunction expansion [11]. More recently, Chen [7] investigated the singularities of thermoelectroelastic fields near the apex of a piezoelectric bonded wedge based on the generalized Lekhnitskii formulation and Mellin transform.

From the above review, it can be seen that most of the previous studies have been devoted to cases of one or two materials and there is very limited work involving multi-material junctions, especially piezoelectric composite wedges. With growth in the number of materials, singularity analysis of electromechanical fields may become increasingly complex and difficult within the framework of Euclidean space. To address this problem, Zhang and Zhong [12], in their study of stress singularity near the crack in an elastic multi-material junction with arbitrary vertex angles, developed a novel symplectic approach based on the Hamiltonian system rather than the conventional Lagrangian system in Euclidean space. The Hamiltonian system used here was introduced to elasticity theory by Zhong [13] in 1995 and is based on the theory of analogy between computational structural mechanics and optimal control, later detailed in Yao and Zhong's book [14]. With the Hamiltonian system, the symplectic eigenfunction expansion and the separation of variables are used to solve elastic problems within the symplectic geometry space rather than Euclidean space [15]. This feature allows the analytical solution of some elastic problems to be obtained more easily and rationally than traditional methods, such as the semiinverse method. Although the Hamiltonian system theory has been widely applied to elastic mechanics problems, applications of this new approach to multi-field problems, such as elastic stress fields and electric fields, have been rarely reported in the literature. To the authors' knowledge, there are only a few studies dealing with piezoelectric and magnetoelectroelastic materials [16-18]. Bian [16] derived the basic equations of electro-mechanical coupling problems in the Hamiltonian system for a piezoelectric strip. Yao [17] qualitatively analyzed the symplectic solution system and Saint-Venant principle for a magnetoelectroelastic strip under antiplane deformation. Wang and Qin [18] extended Bian's results to the case of plane deformation in a magnetoelectroelastic strip. However, none of these studies provides examples to show the practical application and validity of this new method.

The main object of this paper is to develop a symplectic formulation of the Hamiltonian system for analyzing singular behaviour near the apex of a multimaterial piezoelectric wedge under antiplane deformation. For simplicity, the polar coordinate system is employed. After a variable transformation to the polar coordinate system, the radial coordinate is analogous to the 'time coordinate', and then the hoop direction becomes the transverse direction. Displacement and electric potential are treated as state vectors, while shear stress and electric displacement in the radial direction are treated as dual vectors; then, the governing differential equations can be converted into the Hamiltonian system by a differential 
equation approach or a modified variational principle approach. Equations for the dual state vector mentioned above can, then, be obtained. By means of a coordinate transform as well as using the boundary conditions and continuity conditions at the interface, the singularity order for multi-dissimilar piezoelectric and piezoelectric-elastic composite wedges can be determined. Some numerical examples are considered to demonstrate the potential applications and validity of the proposed model to singularity analysis of multi-field materials. The singularity results for the case of piezoelectric wedges with one or two dissimilar materials are shown to be identical with those obtained by other methods [10]. The study also shows that the order of singularity depends strongly on the piezoelectric constant, the geometry and shear modulus of the wedge.

\section{Basic equations and their Hamiltonian system}

\subsection{Basic equations}

In the case of anti-plane shear deformation involving out-of-plane displacement $w$ and in-plane electric fields only, the constitutive equations become [19]:

$$
\left\{\begin{array}{c}
\tau_{\theta z} \\
\tau_{r z} \\
D_{r} \\
D_{\theta}
\end{array}\right\}=\left[\begin{array}{cccc}
c_{44} & 0 & 0 & -e_{15} \\
0 & c_{44} & -e_{15} & 0 \\
0 & e_{15} & \varepsilon_{11} & 0 \\
e_{15} & 0 & 0 & \varepsilon_{11}
\end{array}\right]\left\{\begin{array}{c}
\gamma_{\theta z} \\
\gamma_{r z} \\
E_{r} \\
E_{\theta}
\end{array}\right\}
$$

in which $\tau_{i j}$ are the shear stresses, $\gamma_{i j}$ are shear strains, $D_{i}$ and $E_{i}(i=r, \theta, j=z)$ are electric displacements and electric fields, respectively, $c_{44}$ is the elastic modulus at the constant electric field, $e_{15}$ is the piezoelectric constant and $\varepsilon_{11}$ the dielectric permittivity at constant strains.

The corresponding equilibrium equations and Maxwell's equation are:

$$
\begin{aligned}
& \frac{\partial\left(r \tau_{r z}\right)}{\partial r}+\frac{\partial \tau_{\theta z}}{\partial \theta}=0, \\
& \frac{\partial\left(r D_{r}\right)}{\partial r}+\frac{\partial D_{\theta}}{\partial \theta}=0 .
\end{aligned}
$$

The shear strains and electric fields are given by:

$$
\begin{aligned}
& \gamma_{r z}=\frac{\partial w}{\partial r}, \quad \gamma_{\theta z}=\frac{1}{r} \frac{\partial w}{\partial \theta} \\
& E_{r}=-\frac{\partial \varphi}{\partial r}, \quad E_{\theta}=-\frac{1}{r} \frac{\partial \varphi}{\partial \theta}
\end{aligned}
$$

where $w$ and $\varphi$ are the displacement and electric potential, respectively.

\subsection{Hamiltonian system by differential equation approach}

There are several ways to obtain the Hamiltonian system for an elastic or multi-field material system. In this section, we show how to derive the Hamiltonian system using differential equation methods, a typical approach among the existing methods. 
To do this, let $r, \theta$ represent radial and transverse coordinates, respectively.

Then, introduce the dual vectors $\boldsymbol{q}$ and $\boldsymbol{p}$ :

$$
\boldsymbol{q}=\left\{\begin{array}{c}
w \\
\varphi
\end{array}\right\}, \quad \boldsymbol{p}=\left\{\begin{array}{c}
S_{r} \\
S D_{r}
\end{array}\right\}
$$

which are required in the Hamiltonian system, where

$$
S_{r}=r \tau_{r z}, S D_{r}=r D_{r}
$$

Furthermore, to convert variables and equations from Euclidean space to symplectic geometry space, introduce a generalized time variable $\xi$ such that

$$
\xi=\ln (r)
$$

Since $\xi$ is now a generalized time variable, the symbol '. ' is used to represent the differential with respect to $\xi$.

Making use of equations (2), (5) and (6) we have:

$$
\frac{\partial S_{r}}{\partial r}=\frac{1}{r} \frac{\partial S_{r}}{\partial \xi}=\frac{1}{r} \dot{S}_{r}=-\frac{\partial \tau_{\theta z}}{\partial \theta}=-\frac{1}{r}\left[c_{44} \frac{\partial^{2} w}{\partial \theta^{2}}+e_{15} \frac{\partial^{2} \varphi}{\partial \theta^{2}}\right]
$$

Equation (7) can be further written in the form of the first equation of equation (8), and the expression of $S \dot{D}_{r}$ can be obtained in a similar way as:

$$
\dot{S}_{r}=-c_{44} \frac{\partial^{2} w}{\partial \theta^{2}}-e_{15} \frac{\partial^{2} \varphi}{\partial \theta^{2}}, \quad S \dot{D}_{r}=-e_{5} \frac{\partial^{2} w}{\partial \theta^{2}}+\varepsilon_{11} \frac{\partial^{2} \varphi}{\partial \theta^{2}}
$$

Considering equations (1) and (6), the variables $S_{r}$ and $S D_{r}$ can be expressed as:

$$
S_{r}=c_{44} \dot{w}+e_{15} \dot{\varphi}, \quad S D_{r}=e_{15} \dot{w}-\varepsilon_{11} \dot{\varphi}
$$

Solving equation (9) for $\dot{w}$ and $\dot{\varphi}$, yields:

$$
\dot{w}=\frac{\varepsilon_{11}}{\Delta_{1}} S_{r}+\frac{e_{15}}{\Delta_{1}} S D_{r}, \quad \dot{\varphi}=\frac{e_{15}}{\Delta_{1}} S_{r}-\frac{c_{44}}{\Delta_{1}} S D_{r}
$$

in which

$$
\Delta_{1}=e_{15}^{2}+c_{44} \varepsilon_{11}
$$

The combination of equations (8) and (10) provides following matrix equation:

$$
\left\{\begin{array}{c}
\dot{w} \\
\dot{\varphi} \\
\dot{S}_{r} \\
\dot{S}_{r}
\end{array}\right\}=\left[\begin{array}{cccc}
0 & 0 & \frac{\varepsilon_{11}}{\Delta_{1}} & \frac{e_{15}}{\Delta_{1}} \\
0 & 0 & \frac{e_{15}}{\Delta_{1}} & -\frac{c_{44}}{\Delta_{1}} \\
-c_{44} \frac{\partial^{2}}{\partial \theta^{2}} & -e_{15} \frac{\partial^{2}}{\partial \theta^{2}} & 0 & 0 \\
-e_{15} \frac{\partial^{2}}{\partial \theta^{2}} & \varepsilon_{11} \frac{\partial^{2}}{\partial \theta^{2}} & 0 & 0
\end{array}\right]\left\{\begin{array}{c}
w \\
\varphi \\
S_{r} \\
S D_{r}
\end{array}\right\}
$$


Furthermore, using the notation

$$
v=\left\{\begin{array}{l}
q \\
p
\end{array}\right\}
$$

Equation (12) can be simplified as:

$$
\dot{v}=H v
$$

in which

$$
\boldsymbol{H}=\left[\begin{array}{cccc}
0 & 0 & \frac{\varepsilon_{11}}{\Delta_{1}} & \frac{e_{15}}{\Delta_{1}} \\
0 & 0 & \frac{e_{15}}{\Delta_{1}} & -\frac{c_{44}}{\Delta_{1}} \\
-c_{44} \frac{\partial^{2}}{\partial \theta^{2}} & -e_{15} \frac{\partial^{2}}{\partial \theta^{2}} & 0 & 0 \\
-e_{15} \frac{\partial^{2}}{\partial \theta^{2}} & \varepsilon_{11} \frac{\partial^{2}}{\partial \theta^{2}} & 0 & 0
\end{array}\right]
$$

Equation (14) is the Hamiltonian dual equation. To prove that $\boldsymbol{H}$ is a Hamiltonian operator matrix, a rotational exchange operator matrix $\boldsymbol{J}$ is introduced as follows:

$$
\boldsymbol{J}=\left[\begin{array}{cc}
0 & \boldsymbol{I}_{2} \\
-\boldsymbol{I}_{2} & 0
\end{array}\right], \quad \boldsymbol{J}^{2}=\left[\begin{array}{cc}
-\boldsymbol{I}_{2} & 0 \\
0 & -\boldsymbol{I}_{2}
\end{array}\right], \quad \boldsymbol{J}^{-1}=-\boldsymbol{J}=\boldsymbol{J}^{T}
$$

where $\boldsymbol{I}_{2}$ is the order identity matrix. With the notation $\boldsymbol{J}$, it is easy to prove that $\boldsymbol{H}$ satisfies the following relation (see Appendix A for details of the proof):

$$
\left\langle\boldsymbol{v}_{1}^{T}, \boldsymbol{H} \boldsymbol{v}_{2}\right\rangle=\left\langle\boldsymbol{v}_{2}^{T}, \boldsymbol{H} \boldsymbol{v}_{1}\right\rangle
$$

where

$$
\left\langle\boldsymbol{v}_{1}^{T}, \boldsymbol{H} \boldsymbol{v}_{2}\right\rangle=\int_{\theta_{1}}^{\theta_{2}} \boldsymbol{v}_{1}^{T} \boldsymbol{J} \boldsymbol{H} \boldsymbol{v}_{2} d \theta
$$

Then, according to theory of the symplectic geometry [13, 14], $\boldsymbol{H}$ is a Hamiltonian operator matrix.

\subsection{Hamiltonian system by variational principle approach}

In section 2.1, we derived a Hamiltonian system using a differential equation approach. The same Hamiltonian system for the antiplane problem of a piezoelectric wedge can also be obtained using a variational principle approach. To illustrate this approach, consider following constitutive equation:

$$
\left\{\begin{array}{c}
\gamma_{\theta z} \\
\gamma_{r z} \\
E_{r} \\
E_{\theta}
\end{array}\right\}=\left[\begin{array}{cccc}
s_{44} & 0 & 0 & g_{15} \\
0 & s_{44} & g_{15} & 0 \\
0 & -g_{15} & \beta_{11} & 0 \\
-g_{15} & 0 & 0 & \beta_{11}
\end{array}\right]\left\{\begin{array}{c}
\tau_{\theta z} \\
\tau_{r z} \\
D_{r} \\
D_{\theta}
\end{array}\right\}
$$


where the constants $s_{44}, g_{15} g_{15}$ and $\beta_{11}$ are defined by the relations:

$$
s_{44}=\frac{\varepsilon_{11}}{\Delta_{1}}, \quad g_{15}=\frac{e_{15}}{\Delta_{1}}, \quad \beta_{11}=\frac{c_{44}}{\Delta_{1}}
$$

Based on the constitutive relation (19), the modified Hellinger-Reissner generalized variational principle can be stated as follows:

$$
\begin{aligned}
& \delta\left\{\int _ { r _ { 1 } } ^ { r _ { 2 } } \int _ { \theta _ { 1 } } ^ { \theta _ { 2 } } \left\{\tau_{r z} \frac{\partial w}{\partial r}+\tau_{\theta z} \frac{1}{r} \frac{\partial w}{\partial \theta}+D_{r} \frac{\partial \varphi}{\partial r}+D_{\theta} \frac{1}{r} \frac{\partial \varphi}{\partial \theta}-\left[\frac{1}{2} S_{44} \tau_{\theta z}^{2}+\frac{1}{2} S_{44} \tau_{r z}^{2}-\frac{1}{2} \beta_{11} D_{r}^{2}-\frac{1}{2} \beta_{11} D_{\theta}^{2}\right.\right.\right. \\
& \left.\left.-g_{15} D_{\theta} \tau_{\theta z}-g_{15} \tau_{r z} D_{r}\right]\right\} r d r d \theta=0
\end{aligned}
$$

Making use of the variable transformation equation (4), the variational equality (21) can be further written as:

$$
\begin{aligned}
& \delta\left\{\int _ { \xi _ { 1 } } ^ { \xi _ { 2 } } \int _ { \theta _ { 1 } } ^ { \theta _ { 2 } } \left\{S_{r} \frac{\partial w}{\partial \xi}+S_{\theta} \frac{\partial w}{\partial \theta}+S D_{r} \frac{\partial \varphi}{\partial \xi}+S D_{\theta} \frac{\partial \varphi}{\partial \theta}-\left[\frac{1}{2} S_{44} S_{r}^{2}+\frac{1}{2} S_{44} S_{\theta}^{2}-\frac{1}{2} \beta_{11} S D_{r}^{2}-\frac{1}{2} \beta_{11} S D_{\theta}^{2}\right.\right.\right. \\
& \left.\left.+g_{15} S D_{\theta} S_{\theta}+g_{15} S D_{r} S_{r}\right]\right\} d \xi d \theta=0
\end{aligned}
$$

where

$$
\xi_{1}=\ln r_{1}, \xi_{2}=\ln r_{2}, S_{\theta}=r \tau_{\theta z}, S D_{\theta}=r D_{\theta}
$$

Taking variation with respect to $S_{\theta}$ and $S D_{\theta}$, equation (22) leads to:

$$
S_{\theta}=\frac{1}{\Delta_{2}}\left(\beta_{11} \frac{\partial w}{\partial \theta}+g_{15} \frac{\partial \varphi}{\partial \theta}\right), \quad S D_{\theta}=\frac{1}{\Delta_{2}}\left(g_{15} \frac{\partial w}{\partial \theta}-S_{44} \frac{\partial \varphi}{\partial \theta}\right)
$$

in which

$$
\Delta_{2}=g_{15}^{2}+s_{44} \beta_{11}
$$

Substituting equation (24) into equation (22) we can obtain the Hamiltonian mixed energy variational principle as follows:

$$
\begin{aligned}
& \delta \int_{\xi_{1}}^{\xi_{2}} \int_{\theta_{1}}^{\theta_{2}}\left\{S_{r} \frac{\partial w}{\partial \xi}+S D_{r} \frac{\partial \varphi}{\partial \xi}-\frac{1}{2} S_{44} S_{r}^{2}+\frac{1}{2} \beta_{11} S D_{r}^{2}-g_{15} S D_{r} S_{r}+\frac{1}{2} \frac{1}{\Delta_{2}} \beta_{11}\left(\frac{\partial w}{\partial \theta}\right)^{2}\right. \\
& \left.-\frac{1}{2} \frac{1}{\Delta_{2}} S_{44}\left(\frac{\partial \varphi}{\partial \theta}\right)^{2}+\frac{1}{\Delta_{2}} g_{15} \frac{\partial w}{\partial \theta} \frac{\partial \varphi}{\partial \theta}\right\} d \xi d \theta=0
\end{aligned}
$$

Making use of equation (4), equation (26) can be further simplified as:

$$
\delta \int_{\xi_{1}}^{\xi_{2}} \int_{\theta_{1}}^{\theta_{2}}\left[\boldsymbol{p}^{T} \dot{\boldsymbol{q}}-\Re(\boldsymbol{q}, \boldsymbol{p})\right] d \xi d \theta=0
$$

where $\Re(\boldsymbol{q}, \boldsymbol{p})$ is the Hamiltonian function defined by:

$$
\mathfrak{R}(\boldsymbol{q}, \boldsymbol{p})=-\frac{1}{2} \boldsymbol{q}^{T} \boldsymbol{B} \boldsymbol{q}+\frac{1}{2} \boldsymbol{p}^{T} \boldsymbol{D} \boldsymbol{p}
$$


in which

$$
\boldsymbol{B}=\left[\begin{array}{cc}
-\frac{\beta_{11}}{\Delta_{2}} \frac{\partial^{2}}{\partial \theta^{2}} & -\frac{g_{15}}{\Delta_{2}} \frac{\partial^{2}}{\partial \theta^{2}} \\
-\frac{g_{15}}{\Delta_{2}} \frac{\partial^{2}}{\partial \theta^{2}} & \frac{s_{44}}{\Delta_{2}} \frac{\partial^{2}}{\partial \theta^{2}}
\end{array}\right], \quad \boldsymbol{D}=\left[\begin{array}{cc}
s_{44} & g_{15} \\
g_{15} & -\beta_{11}
\end{array}\right]
$$

In the derivation of equation (27), homogeneous boundary conditions (see Appendix A) have been used.

From equation (27) the following equations can be obtained:

$$
\begin{aligned}
& \dot{\boldsymbol{q}}=\boldsymbol{D p} \\
& \dot{\boldsymbol{p}}=\boldsymbol{B} \boldsymbol{q}
\end{aligned}
$$

Using the definition of $v$ in equation (13), equation (30) can be rewritten in the form:

$$
\dot{v}=H^{*} v
$$

in which

$$
\boldsymbol{H}^{*}=\left(\begin{array}{cc}
0 & \boldsymbol{D} \\
\boldsymbol{B} & 0
\end{array}\right)
$$

where $\boldsymbol{H}^{*}$ is used to distinguish the matrix $\boldsymbol{H}$ in equation (14). Making use of the relation (20), it can be found that $\boldsymbol{H}^{*}$ in equation (32) is the same as $\boldsymbol{H}$ in equation (15). Therefore, equations (31) and (14) are identical.

\section{Basic eigenvalues and singularity of stress and electric fields}

In section 2, the dual state vector equations (14) and (31) were derived using the differential equation method and the variational principle approach, respectively. In this section, applications of the proposed Hamiltonian model to analyzing the eigenvalues of the Hamiltonian operator matrix, which are associated with the singularity behaviour of a piezoelectric wedge, are discussed.

Noting that equation (14) can be solved by the separation of the variable and the symplectic eigenfunction expansion, one can assume $\boldsymbol{v}$ in the form:

$$
\boldsymbol{v}(\xi, \theta)=\boldsymbol{\kappa}(\xi) \psi(\theta)
$$

in which $\boldsymbol{\kappa}(\xi), \boldsymbol{\psi}(\theta)$ are two functions of $\xi, \theta$ respectively.

Substituting equation (33) into equation (14) yields the solution for $\boldsymbol{\kappa}(\xi)$ as:

$$
\boldsymbol{\kappa}(\xi)=e^{\mu \xi}
$$

and the eigenvalue equation:

$$
\boldsymbol{H} \psi=\mu \boldsymbol{\psi}
$$


in which $\mu$ is an eigenvalue of the Hamiltonian operator matrix and $\psi$ is given by:

$$
\psi=\left\{\begin{array}{l}
\boldsymbol{q}(\theta) \\
\boldsymbol{p}(\theta)
\end{array}\right\}
$$

Thus, we have:

$$
\boldsymbol{v}=e^{\mu \xi} \psi(\theta)
$$

It should be mentioned that the eigenvalues of the Hamiltonian operator matrix have following property [13]: if $\mu_{i}$ is an eigenvalue of equation (35), then $-\mu_{i}$ is also an eigenvalue of equation (35). With this property, all eigenvalues of $\boldsymbol{H}$ can be subdivided into the following three groups:

(a) $\mu_{i}, \quad \operatorname{Re}\left(\mu_{i}\right)>0$ or $\operatorname{Im}\left(\mu_{i}\right)>0$ (if $\left.\operatorname{Re}\left(\mu_{i}\right)=0\right) \quad i=1,2$,

(b) $\mu_{-i}=-\mu_{i}$

(c) $\mu=0$

Their corresponding eigenfunction-vectors are written as $\psi_{+i}, \psi_{-i}$ and $\psi_{0}$. Following the procedure in [13], it is easy to prove that $\psi_{+i}$ and $\psi_{-i}$ are of adjoint symplectic orthonormalization, that is,

$$
\begin{aligned}
& \left\langle\boldsymbol{\psi}_{+i}^{T}, \boldsymbol{J}, \boldsymbol{\psi}_{-j}\right\rangle=\delta_{i j}, \quad\left\langle\boldsymbol{\psi}_{-i}^{T}, \boldsymbol{J}, \boldsymbol{\psi}_{+j}\right\rangle=-\delta_{i j}, \\
& \left\langle\boldsymbol{\psi}_{+i}^{T}, \boldsymbol{J}, \boldsymbol{\psi}_{+j}\right\rangle=0, \quad\left\langle\boldsymbol{\psi}_{-i}^{T}, \boldsymbol{J}, \boldsymbol{\psi}_{-j}\right\rangle=0
\end{aligned}
$$

in which

$$
\left\langle\psi_{i}^{T}, \boldsymbol{J}, \psi_{j}\right\rangle=\int_{\theta_{1}}^{\theta_{2}} \psi_{i}^{T} \boldsymbol{J} \psi_{j} d \theta
$$

Equation (39) implies that $\psi_{i}$ satisfies the homogeneous boundary conditions at $\theta=\theta_{1}, \theta_{2}$.

Proof: Considering two eigenfunction-vectors, $\boldsymbol{\psi}_{i}, \boldsymbol{\psi}_{j}$, we have from equation (35) that

$$
\boldsymbol{H} \boldsymbol{\psi}_{i}=\mu_{i} \boldsymbol{\psi}_{i}, \boldsymbol{H} \boldsymbol{\psi}_{j}=\mu_{j} \boldsymbol{\psi}_{j}
$$

in which $\boldsymbol{H}$ satisfies the following relation:

$$
\boldsymbol{H}^{T} \cdot\left(\boldsymbol{J} \boldsymbol{\psi}_{i}\right)=-\mu_{i} \boldsymbol{J} \boldsymbol{\psi}_{i}
$$

Multiplying $\boldsymbol{\psi}_{j}^{T}$ on both sides of equation (41) and integrating it across the transverse section, we can obtain the following equation:

$$
\left\langle\boldsymbol{\psi}_{j}^{T}, \boldsymbol{H}^{T}, J \boldsymbol{\psi}_{i}\right\rangle=-\left\langle\boldsymbol{\psi}_{i}^{T}, \boldsymbol{J} \boldsymbol{H}, \boldsymbol{\psi}_{j}\right\rangle=-\mu_{i}\left\langle\boldsymbol{\psi}_{j}^{T}, \boldsymbol{J}, \boldsymbol{\psi}_{i}\right\rangle=\mu_{i}\left\langle\boldsymbol{\psi}_{i}^{T}, \boldsymbol{J}, \boldsymbol{\psi}_{j}\right\rangle
$$

in which equation (16) has been used. Similarly, it is easy to show that the following relation holds true:

$$
\left\langle\boldsymbol{\psi}_{i}^{T}, \boldsymbol{J} \boldsymbol{H}, \boldsymbol{\psi}_{j}\right\rangle=\mu_{j}\left\langle\boldsymbol{\psi}_{i}^{T}, \boldsymbol{J}, \boldsymbol{\psi}_{j}\right\rangle
$$


Making use of equations (42) and (43), we have:

$$
\left(\mu_{i}+\mu_{j}\right) \cdot\left\langle\psi_{i}^{T}, \boldsymbol{J}, \psi_{j}\right\rangle=0
$$

If $\mu_{i}+\mu_{j} \neq 0$, equation (44) is reduced to:

$$
\left\langle\psi_{i}^{T}, \boldsymbol{J}, \boldsymbol{\psi}_{j}\right\rangle=0
$$

When $\mu_{i}+\mu_{j}=0$, i.e., $j=-i, \psi_{i}$ and $\psi_{-i}$ satisfy the following relation:

$$
\boldsymbol{\psi}_{i}^{T} \boldsymbol{J} \psi_{-i} \neq 0 \quad(i=1,2, \ldots, n)
$$

Performing the orthonormality operation, we obtain:

$$
\boldsymbol{\psi}_{i}^{T} J \boldsymbol{\psi}_{-i}=1, \text { and } / \text { or } \boldsymbol{\psi}_{-i}^{T} J \boldsymbol{\psi}_{i}=-1 \quad(i=1,2, \ldots, n)
$$

This indicates that equation (38) holds true. The adjoint symplectic orthonormalization relation between eigenfunctions has thus been proved.

Since $\psi_{+i}$ and $\psi_{-i}$ are of adjoint symplectic orthonormalization, the state vector $\psi$ can be expressed by the linear combination of the eigenfunction-vectors as follows:

$$
\psi=\sum_{i=1}^{\infty}\left(a_{i} \psi_{i}+b_{i} \psi_{-i}\right)
$$

where $\psi_{i}$ and $\psi_{-i}$ are eigenfunction vectors corresponding to $\mu_{i}$ and $\mu_{-i}$ and $a_{i}, b_{i}$ are coefficients to be determined.

From equations (4) and (37) we can obtain the following expressions:

$$
\begin{gathered}
\left\{\begin{array}{c}
w \\
\varphi
\end{array}\right\}=r^{\mu} \boldsymbol{q}(\theta) \\
\left\{\begin{array}{c}
\tau_{r z} \\
D_{r}
\end{array}\right\}=r^{\mu-1} \boldsymbol{p}(\theta)
\end{gathered}
$$

From equation (50) it can be found that the stresses and electric displacements near the apex of a wedge are proportional to $r^{\mu-1}$; therefore, the singularity order of the stresses and electric displacements is $\operatorname{Re}(\mu)-1$.

It is obvious that the stresses and electric displacements are singular if the real part of $\mu$ is less than 1 , i.e. $\operatorname{Re}(\mu)<1$. For the potential energy to be bounded at the apex of the wedge, it is required that $\operatorname{Re}(\mu)>0$. So we focus our attention on the interval:

$$
0<\operatorname{Re}(\mu)<1
$$

Using the notation of the generalized stress and electric displacement intensity factors $K^{\sigma}$ and $K^{D}$, equation (50) can be rewritten as:

$$
\begin{aligned}
& \tau_{r z}(r, \theta)=K^{\sigma} r^{\lambda^{*}-1} f^{\sigma}(\theta) \\
& D_{r}(r, \theta)=K^{D} r^{\lambda^{*}-1} f^{D}(\theta)
\end{aligned}
$$


where $\lambda^{*}-1$ is the orders of the stress and electric displacement singularity, and $f^{\sigma}(\theta)$ and $f^{D}(\theta)$ are the angular function

From equations (50) and (52) it is easy to see that:

$$
\psi(\theta)=\boldsymbol{K} f(\theta), \lambda^{*}=\mu
$$

in which

$$
\boldsymbol{K}=\left[\begin{array}{cc}
K^{\sigma} & 0 \\
0 & K^{D}
\end{array}\right] \text {, and } f(\theta)=\left\{f^{\sigma}(\theta), f^{D}(\theta)\right\}^{T}
$$

The remaining task is to find the angular function $f(\theta)$ and the generalized stress and electric displacement intensity factor $\mathbf{K}$ (the definition of the generalized stress intensity factors can be found in [11]). Therefore, we need to find eigenvalues of equation (35), which satisfy the condition equation (51). To this end, rewriting equation (35) in terms of its matrix components, we have

$$
\left[\begin{array}{cccc}
-\mu & 0 & \frac{\varepsilon_{11}}{\Delta_{1}} & \frac{e_{15}}{\Delta_{1}} \\
0 & -\mu & \frac{e_{15}}{\Delta_{1}} & -\frac{c_{44}}{\Delta_{1}} \\
-c_{44} \frac{d^{2}}{d \theta^{2}} & -e_{15} \frac{d^{2}}{d \theta^{2}} & -\mu & 0 \\
-e_{15} \frac{d^{2}}{d \theta^{2}} & \varepsilon_{11} \frac{d^{2}}{d \theta^{2}} & 0 & -\mu
\end{array}\right]\left\{\begin{array}{c}
w \\
\varphi \\
S_{r} \\
S D_{r}
\end{array}\right\}=0
$$

The order of singularity in the elastic and electric fields is determined by setting the determinant of the $4 \times 4$ matrix in equation (54) to zero. This is equivalent to:

$$
\left|\begin{array}{cccc}
-\mu & 0 & \frac{\varepsilon_{11}}{\Delta_{1}} & \frac{e_{15}}{\Delta_{1}} \\
0 & -\mu & \frac{e_{15}}{\Delta_{1}} & -\frac{c_{44}}{\Delta_{1}} \\
-c_{44} \lambda^{2} & -e_{15} \lambda^{2} & -\mu & 0 \\
-e_{15} \lambda^{2} & \varepsilon_{11} \lambda^{2} & 0 & -\mu
\end{array}\right|=0
$$

where $\lambda$ is the eigenvalue in $\theta$ direction.

Equation (55) leads to the following equation:

$$
\left(\lambda^{2}+\mu^{2}\right)^{2}=0
$$

Thus, the solutions of $\lambda$ are:

$$
\lambda_{1,2}=\mu i, \lambda_{3,4}=-\mu i
$$

With solution (57), the general expressions of the elastic and electric fields can be expressed as:

$$
\begin{aligned}
w & =A_{1} \cos (\mu \theta)+B_{1} \sin (\mu \theta)+C_{1} \theta \cos (\mu \theta)+D_{1} \theta \sin (\mu \theta), \\
\varphi & =A_{2} \cos (\mu \theta)+B_{2} \sin (\mu \theta)+C_{2} \theta \cos (\mu \theta)+D_{2} \theta \sin (\mu \theta), \\
S_{r} & =A_{3} \cos (\mu \theta)+B_{3} \sin (\mu \theta)+C_{3} \theta \cos (\mu \theta)+D_{3} \theta \sin (\mu \theta), \\
S D_{r} & =A_{4} \cos (\mu \theta)+B_{4} \sin (\mu \theta)+C_{4} \theta \cos (\mu \theta)+D_{4} \theta \sin (\mu \theta) .
\end{aligned}
$$


where $A_{i}, B_{i}, C_{i}, D_{i}(i=1-4)$ are unknown constants to be determined.

Substituting equation (58) into equation (54) yields the following relationships among the four unknown constants (the equations of these constants are listed in Appendix B):

$$
\begin{aligned}
& A_{3}=\mu\left(c_{44} A_{1}+e_{15} A_{2}\right), \quad A_{4}=\mu\left(e_{15} A_{1}-\varepsilon_{11} A_{2}\right), \\
& B_{3}=\mu\left(c_{44} B_{1}+e_{15} B_{2}\right), \quad B_{4}=\mu\left(e_{15} B_{1}-\varepsilon_{11} B_{2}\right), \\
& D_{i}=C_{i}=0 \quad(i=1-4) .
\end{aligned}
$$

Then, equation (58) can be rewritten as:

$$
\begin{aligned}
\left\{\begin{array}{l}
w \\
\varphi
\end{array}\right\} & =\left[\begin{array}{ll}
A_{1} & B_{1} \\
A_{2} & B_{2}
\end{array}\right]\left\{\begin{array}{l}
\cos (\mu \theta) \\
\sin (\mu \theta)
\end{array}\right\}, \\
\left\{\begin{array}{c}
S_{r} \\
S D_{r}
\end{array}\right\} & =\mu\left[\begin{array}{ll}
c_{44} & e_{15} \\
e_{15} & -\varepsilon_{11}
\end{array}\right]\left\{\begin{array}{l}
A_{1} \\
A_{2}
\end{array}\right\} \cos (\mu \theta)+\mu\left[\begin{array}{cc}
c_{44} & e_{15} \\
e_{15} & -\varepsilon_{11}
\end{array}\right]\left\{\begin{array}{l}
B_{1} \\
B_{2}
\end{array}\right\} \sin (\mu \theta) .
\end{aligned}
$$

From equations (1) and (60) we have:

$$
\left\{\begin{array}{c}
S_{\theta} \\
S D_{\theta}
\end{array}\right\}=\mu\left[\begin{array}{cc}
c_{44} & e_{15} \\
e_{15} & -\varepsilon_{11}
\end{array}\right]\left[\begin{array}{ll}
A_{1} & B_{1} \\
A_{2} & B_{2}
\end{array}\right]\left\{\begin{array}{c}
-\sin (\mu \theta) \\
\cos (\mu \theta)
\end{array}\right\}
$$

To obtain explicit expression of the four unknown constants, consider a piezoelectric wedge as shown in figure 1 . The conditions at the boundary edges are assumed to be free of traction and electrically insulated:

$$
\tau_{\theta z}(r, \alpha)=\tau_{\theta z}(r,-\beta)=D_{\theta}(r, \alpha)=D_{\theta}(r,-\beta)=0
$$

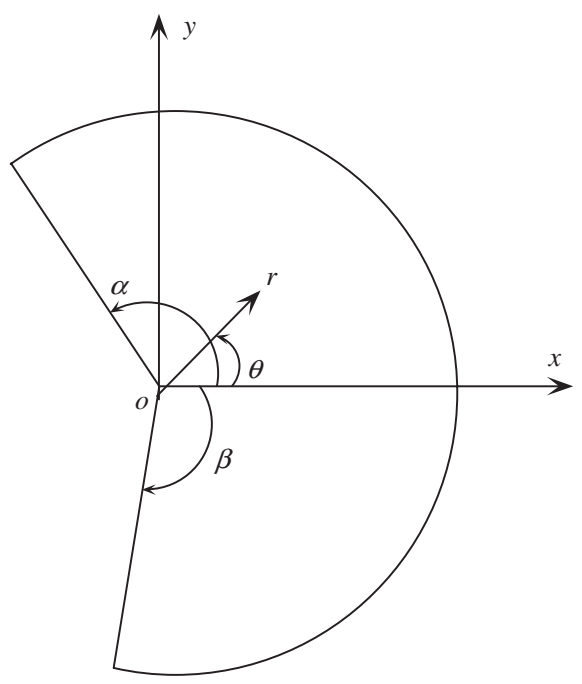

Figure 1. Piezoelectric wedge. 
Substituting equations (60) and (61) into equation (62) yields:

$$
\left[\begin{array}{cccc}
-c_{44} \sin (\mu \alpha) & -e_{15} \sin (\mu \alpha) & c_{44} \cos (\mu \alpha) & c_{44} \cos (\mu \alpha) \\
-e_{15} \sin (\mu \alpha) & \varepsilon_{11} \sin (\mu \alpha) & e_{15} \cos (\mu \alpha) & -\varepsilon_{11} \cos (\mu \alpha) \\
c_{44} \sin (\mu \beta) & e_{15} \sin (\mu \beta) & c_{44} \cos (\mu \beta) & e_{15} \cos (\mu \beta) \\
e_{15} \sin (\mu \beta) & -\varepsilon_{11} \sin (\mu \beta) & e_{15} \cos (\mu \beta) & -\varepsilon_{11} \cos (\mu \beta)
\end{array}\right]\left\{\begin{array}{l}
A_{1} \\
A_{2} \\
B_{1} \\
B_{2}
\end{array}\right\}=0
$$

The condition for the existence of non-zero solutions of $\left\{\begin{array}{llll}A_{1} & A_{2} & B_{1} & B_{2}\end{array}\right\}^{T}$ is the determinant of the coefficients matrix being zero, which leads to the following equation:

$$
\left(c_{44} \varepsilon_{11}+e_{15}^{2}\right)^{2} \sin ^{2}(\mu(\alpha+\beta))=0
$$

If $\alpha=\beta=\pi$, we have $\mu=1 / 2$, and the order of singularity is $-1 / 2$, which is the classical square-root singularity for a semi-infinite crack. This result also verifies the validity of this method for the case of a semi-infinite crack.

Consider now a piezoelectric half plane, i.e. $\alpha=\beta=\pi / 2$. It can be easily found that no root of equation (64) can satisfy the condition $0<\operatorname{Re}(\mu)<1$. Therefore, there is no singularity for the piezoelectric half plane under the homogeneous boundary condition. The same results have also been obtained for a piezoelectric half plane under anti-plane deformation [11]. We also note that the singularity disappears for $\alpha+\beta \leq 180^{\circ}$. For $180^{\circ}<\alpha+\beta<360^{\circ}$, the variation of the order of singularity with $\alpha+\beta$ is plotted in figure 2. It can be seen that for a homogeneous piezoelectric wedge, the order of singularity depends on the value of $\alpha+\beta$ only.

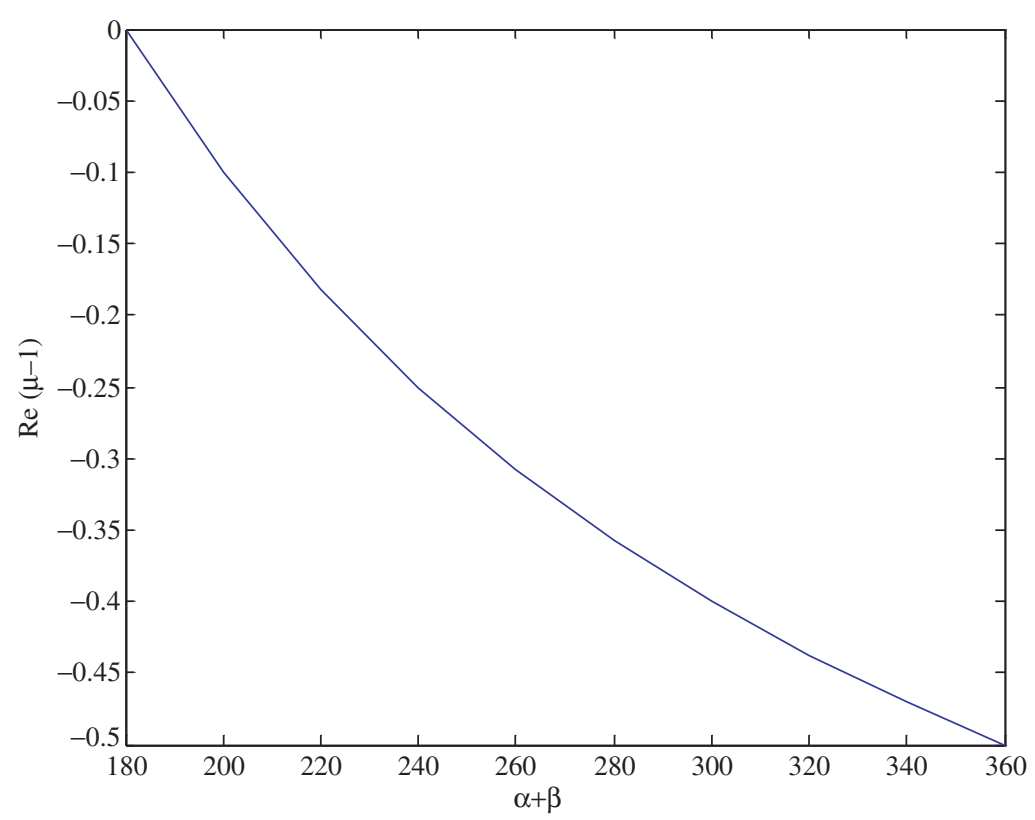

Figure 2. Variation of order of singularity with $\alpha+\beta$ for a piezoelectric wedge. 
It should be mentioned here that for the sake of simplicity only one type of boundary condition on the edges, given as equation (62), is considered. However, for other types of boundary conditions such as clamped $(w=0)$ and electrically open $(\phi=0)$, this procedure is also applicable and the results can be obtained in a similar way.

\section{Piezoelectric bimaterial wedge}

For a piezoelectric bimaterial wedges as shown in figure 3, the boundary conditions are as follows:

$$
\tau_{\theta z}^{(1)}(r, \alpha)=\tau_{\theta z}^{(2)}(r,-\beta)=D_{\theta}^{(1)}(r, \alpha)=D_{\theta}^{(2)}(r,-\beta)=0
$$

If the bimaterials are rigidly bonded at the interface, the continuity conditions on the interface are:

$$
\begin{aligned}
\tau_{\theta z}^{(1)}(r, 0) & =\tau_{\theta z}^{(2)}(r, 0), \quad w^{(1)}(r, 0)=w^{(2)}(r, 0), \\
D_{\theta}^{(1)}(r, 0) & =D_{\theta}^{(2)}(r, 0), \quad E_{r}^{(1)}(r, 0)=E_{r}^{(2)}(r, 0) .
\end{aligned}
$$

in which superscripts (1) and (2) denote materials 1 and 2, respectively.

As shown in figure 3, each material can be viewed as a homogeneous wedge. Using the general solution (60) of a homogeneous piezoelectric wedge and the continuity condition at its interface, we can easily obtain the solution for a bimaterial wedge. Keeping this in mind, substituting the solutions (60) and (61) for each material into equation (66) yields the relationship between the unknown constants:

$$
\left\{\begin{array}{l}
B_{1}^{(2)} \\
B_{2}^{(2)}
\end{array}\right\}=\left[\begin{array}{ll}
a_{11} & a_{12} \\
a_{21} & a_{22}
\end{array}\right]\left\{\begin{array}{l}
B_{1}^{(1)} \\
B_{2}^{(1)}
\end{array}\right\}
$$

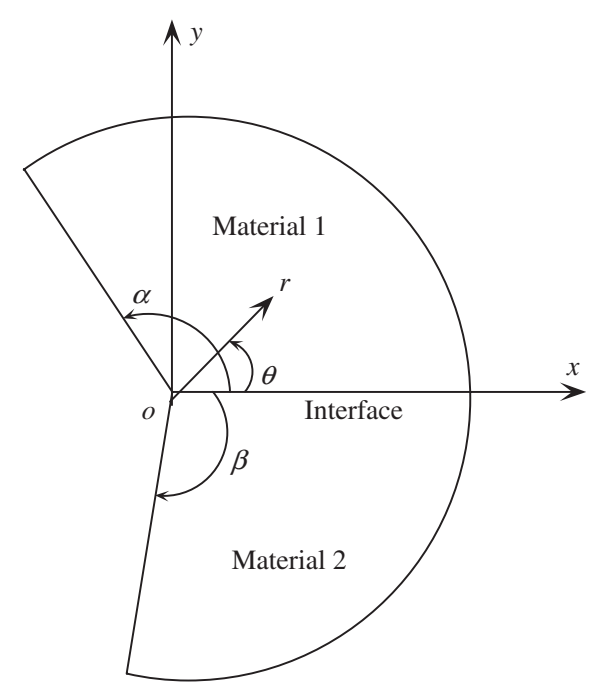

Figure 3. Piezoelectric bimaterial wedge. 
in which

$$
\begin{array}{ll}
a_{11}=\frac{1}{\Delta_{3}}\left(\varepsilon_{11}^{(2)} c_{44}^{(1)}+e_{15}^{(2)} e_{15}^{(1)}\right), & a_{12}=\frac{1}{\Delta_{3}}\left(\varepsilon_{11}^{(2)} e_{15}^{(1)}-e_{15}^{(2)} \varepsilon_{11}^{(1)}\right), \\
a_{21}=\frac{1}{\Delta_{3}}\left(e_{15}^{(2)} c_{44}^{(1)}-c_{44}^{(2)} e_{15}^{(1)}\right), & a_{22}=\frac{1}{\Delta_{3}}\left(e_{15}^{(2)} e_{15}^{(1)}+c_{44}^{(2)} \varepsilon_{11}^{(1)}\right),
\end{array}
$$

where

$$
\Delta_{3}=c_{44}^{(2)} \varepsilon_{11}^{(2)}+e_{15}^{(2) 2}
$$

Using the relation (67) and substituting the solutions (60) and (61) into equation (65) leads to:

$$
\left[\begin{array}{cccc}
-c_{44}^{(1)} \sin (\mu \alpha) & -e_{15}^{(1)} \sin (\mu \alpha) & c_{44}^{(1)} \cos (\mu \alpha) & e_{15}^{(1)} \cos (\mu \alpha) \\
-e_{15}^{(1)} \sin (\mu \alpha) & \varepsilon_{11}^{(1)} \sin (\mu \alpha) & e_{15}^{(1)} \cos (\mu \alpha) & -\varepsilon_{11}^{(1)} \cos (\mu \alpha) \\
c_{44}^{(2)} \sin (\mu \beta) & e_{15}^{(2)} \sin (\mu \beta) & {\left[c_{44}^{(2)} a_{11}+e_{15}^{(2)} a_{21}\right] \cos (\mu \beta)} & {\left[c_{44}^{(2)} a_{12}+e_{15}^{(2)} a_{22}\right] \cos (\mu \beta)} \\
e_{15}^{(2)} \sin (\mu \beta) & -\varepsilon_{11}^{(1)} \sin (\mu \beta) & {\left[e_{15}^{(2)} a_{11}-\varepsilon_{11}^{(2)} a_{21}\right] \cos (\mu \beta)} & {\left[e_{15}^{(2)} a_{12}-\varepsilon_{11}^{(2)} a_{22}\right] \cos (\mu \beta)}
\end{array}\right]\left\{\begin{array}{c}
A_{1}^{(1)} \\
A_{2}^{(1)} \\
B_{1}^{(1)} \\
B_{2}^{(1)}
\end{array}\right\}=0
$$

The non-zero solution of equation (70) requires that:

$$
\begin{aligned}
& \left(c_{44}^{(1)} \varepsilon_{11}^{(1)}+e_{15}^{(1) 2}\right) \sin ^{2}(\mu \alpha) \cos ^{2}(\mu \beta)+\left(c_{44}^{(2)} \varepsilon_{11}^{(1)}+e_{15}^{(2) 2}\right) \sin ^{2}(\mu \beta) \cos ^{2}(\mu \alpha) \\
& +\left(c_{44}^{(1)} \varepsilon_{11}^{(2)}+2 e_{15}^{(1)} e_{15}^{(2)}+c_{44}^{(2)} \varepsilon_{11}^{(1)}\right) \sin (\mu \alpha) \sin (\mu \beta) \cos (\mu \alpha) \cos (\mu \beta)=0
\end{aligned}
$$

or written as:

$$
\sin ^{2}(\mu(\alpha+\beta))+R_{1} \sin ^{2}(\mu(\alpha-\beta))=R_{2} \sin (\mu(\alpha+\beta)) \sin (\mu(\alpha-\beta))
$$

with:

$$
\begin{aligned}
& R_{1}=\frac{A_{11}+A_{22}-A_{12}-A_{21}}{A_{11}+A_{22}+A_{12}+A_{21}}, \quad R_{2}=\frac{2\left(A_{22}-A_{11}\right)}{A_{11}+A_{22}+A_{12}+A_{21}} \\
& A_{11}=e_{15}^{(1)} e_{15}^{(1)}+c_{44}^{(1)} \varepsilon_{11}^{(1)}, \quad A_{12}=e_{15}^{(1)} e_{15}^{(2)}+\varepsilon_{11}^{(1)} c_{44}^{(2)} \\
& A_{21}=e_{15}^{(2)} e_{15}^{(1)}+\varepsilon_{11}^{(2)} c_{44}^{(1)}, \quad A_{22}=e_{15}^{(2)} e_{15}^{(2)}+c_{44}^{(2)} \varepsilon_{11}^{(2)}
\end{aligned}
$$

It is found that equation (72) is exactly the same as those of Chue and Chen [10] and Chen and Chue [11]. The solution of equation (72) is given in detail in [11] for two cases of $\alpha=\beta$ and $\alpha \neq \beta$ to show the singular electro-mechanical field. For an interface crack, i.e. $\alpha=\beta=\pi$, we have $\mu=-1 / 2$, which returns to a classical $-1 / 2$ singularity. For other values of $\alpha$ and $\beta$, equation (72) shows that the order of singularity strongly depends on the geometry and material constants of the two piezoelectric materials. In addition, the angular function and generalized stress and electrical intensity factors $K^{\sigma}$ and $K^{D}$ can also be obtained easily using this method. It should be mentioned, however, that equation (72) derives from the $4 \times 4$ homogeneous equation (70), while in [11], it derives from $8 \times 8$ 
homogeneous equations. Compared to the conventional method [11], the proposed symplectic model can solve the singularity problem more rationally. In particular, with the increase of number of materials, the conventional method will induce a large number of complicated equation systems which may be difficult to be solved theoretically. In contrast, the model developed in this paper solves the problem above with the matrix operations and does not suffer the problem induced by the number of materials. In addition, this model is particularly useful for analyzing problems with local effects, such as the field singularity problem and the SaintVenant decay problem.

\section{Multi-material wedge}

In sections 3 and 4, the theory of the Hamiltonian system was used to develop symplectic models of a piezoelectric wedge and, then, to determine the orders of singularity for both a homogeneous wedge and a bimaterial wedge. In the following, the results obtained are extended to the case of multi-material wedges, including multi-piezoelectric materials and combinations of piezoelectric-elastic materials.

\subsection{Multi-piezoelectric material wedge}

Consider a piezoelectric wedge consisting of multi-piezoelectric material elements as shown in figure 4 , which is similar to the multi-elastic material wedge in [12]. Here, $N$ is the number of material elements. The polar coordinate is again selected

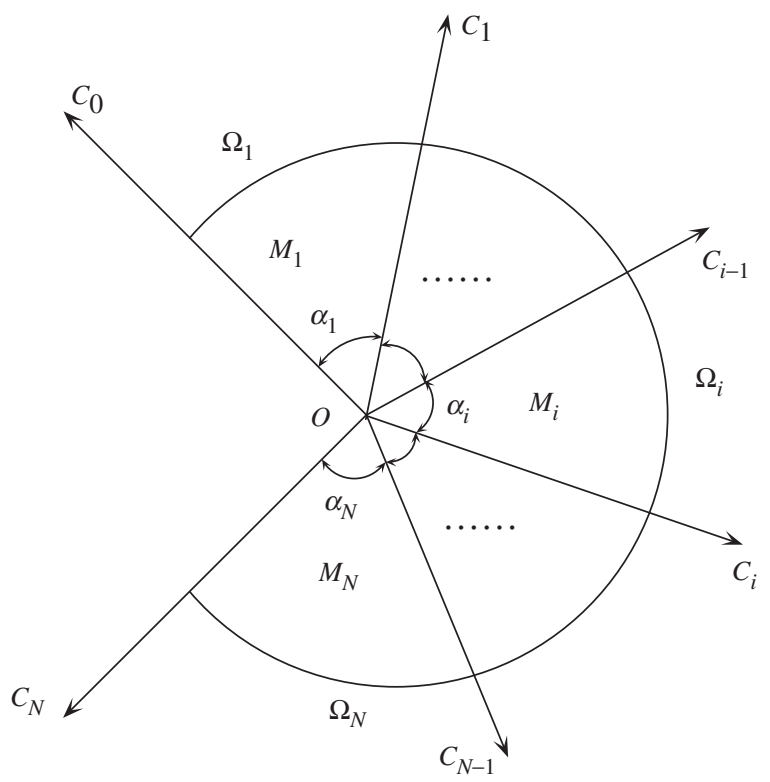

Figure 4. Multi-material wedge. 
for simplicity, and $C_{0}, C_{1}, \ldots, C_{N}$ is adopted to indicate the $0-N$ sub-polar coordinate systems. The domain $\Omega_{i}$ denotes the material element $M_{i}$, and $\alpha_{i}$ is the angle of $M_{i}$.

The continuity conditions on the bonded interface region are:

$$
\begin{aligned}
& \tau_{\theta z}^{(i)}(r, 0)=\tau_{\theta z}^{(i+1)}(r, 0), \quad w^{(i)}(r, 0)=w^{(i+1)}(r, 0), \\
& D_{\theta}^{(i)}(r, 0)=D_{\theta}^{(i+1)}(r, 0), \quad E_{r}^{(i)}(r, 0)=E_{r}^{(i+1)}(r, 0)
\end{aligned}
$$

in which the superscript $i$ runs from 1 to $N-1$, which represents the associated variable defined in the domain $\Omega_{i}$. It should be mentioned that the fields in the two adjacent regions $\Omega_{i}$ and $\Omega_{i+1}$ in equation (74) are written in terms of the coordinate system $C_{i}$ with both of the two regions having $\theta=0$ at the interface. This is for the sake of simplicity.

The boundary conditions of this problem are:

$$
\tau_{\theta z}^{(1)}\left(r, \alpha_{1}\right)=D_{\theta}^{(1)}\left(r, \alpha_{1}\right)=\tau_{\theta z}^{(N)}\left(r,-\alpha_{N}\right)=D_{\theta}^{(N)}\left(r,-\alpha_{N}\right)=0
$$

Note that solution (60) also applies for each single domain $\Omega_{i}$. Thus, substitution of the general solutions (60) for the domain $\Omega_{i}$ and $\Omega_{i+1}$ into equation (74) yields the relationship of the unknown constants for any two adjacent domains as follows:

$$
\left\{F_{i}^{i+1}\right\}=[R(i, i+1)]\left\{F_{i}^{i}\right\}
$$

where

$$
\begin{aligned}
& {[R(i, i+1)]=\left[\begin{array}{cccc}
1 & 0 & 0 & 0 \\
0 & 1 & 0 & 0 \\
0 & 0 & r_{11} & r_{12} \\
0 & 0 & r_{21} & r_{22}
\end{array}\right]}
\end{aligned}
$$

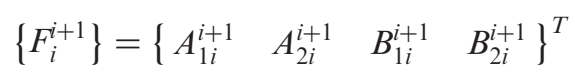

and

$$
\begin{array}{ll}
r_{11}=\frac{1}{\Delta_{r}}\left(\varepsilon_{11}^{(i+1)} c_{44}^{(i)}+e_{15}^{(i+1)} e_{15}^{(i)}\right), & r_{12}=\frac{1}{\Delta_{r}}\left(\varepsilon_{11}^{(i+1)} e_{15}^{(i)}-e_{15}^{(i+1)} \varepsilon_{11}^{(i)}\right), \\
r_{21}=\frac{1}{\Delta_{r}}\left(e_{15}^{(i+1)} c_{44}^{(i)}-c_{44}^{(i+1)} e_{15}^{(i)}\right), & r_{22}=\frac{1}{\Delta_{r}}\left(e_{15}^{(i+1)} e_{15}^{(i)}+c_{44}^{(i+1)} \varepsilon_{11}^{(i)}\right),
\end{array}
$$

with

$$
\Delta_{r}=c_{44}^{(i+1)} \varepsilon_{11}^{(i+1)}+e_{15}^{(i+1) 2}
$$


In equation (76), the subscript $i$ represents the unknown constants expressed in terms of the coordinates $C_{i}$, and the superscripts $i$ and $i+1$ mean the domains $\Omega_{i}, \Omega_{i+1}$, respectively.

In the following, the coordinate transformation is used to find the relationships between the unknown constants in general solutions of each material domain $\Omega_{i}$ in two coordinate systems $C_{i}$ and. $C_{i-1}$ Assuming the equality

$$
\left\{\begin{array}{c}
w_{i}^{i} \\
\varphi_{i}^{i}
\end{array}\right\}=\left\{\begin{array}{c}
w_{i-1}^{i} \\
\varphi_{i-1}^{i}
\end{array}\right\}
$$

and using the relationships among the trigonometric functions, we obtain:

$$
\left\{F_{i}^{i}\right\}=[T(i, i-1)]\left\{F_{i-1}^{i}\right\}
$$

where

$$
[T(i, i-1)]=\left[\begin{array}{cccc}
\cos \left(\mu \alpha_{i}\right) & 0 & -\sin \left(\mu \alpha_{i}\right) & 0 \\
0 & \cos \left(\mu \alpha_{i}\right) & 0 & -\sin \left(\mu \alpha_{i}\right) \\
\sin \left(\mu \alpha_{i}\right) & 0 & \cos \left(\mu \alpha_{i}\right) & 0 \\
0 & \sin \left(\mu \alpha_{i}\right) & 0 & \cos \left(\mu \alpha_{i}\right)
\end{array}\right]
$$

The combination of equations (76) and (82) yields the relationship:

$$
\left\{F_{N-1}^{N}\right\}=\left[T R_{N}\right]\left\{F_{1}^{1}\right\}
$$

where

$$
\left[T R_{N}\right]=\prod_{i=N-1}^{2}\{[R(i, i+1)][T(i, i-1)]\}[R(1,2)]
$$

It can be seen from equation (84) that solutions in any domain can be expressed by four independent unknown constants defined in $\Omega_{1}$. Considering the boundary conditions (75), we obtain:

$$
[M]\left\{F_{1}^{1}\right\}=0
$$

where $[M]$ is a $4 \times 4$ matrix which has a similar form to that in equation (70) (we omit its details here). The existence of a nontrivial solution for $\left\{F_{1}^{1}\right\}$ requires a zero determinant of the coefficients matrix $[M]$ :

$$
\operatorname{det}[M]=0
$$

Then, the solution for $\mu$ can be obtained by solving equation (87) and the order of singularity is again $\operatorname{Re}(\mu)-1$ by considering the condition in equation (51).

It should be mentioned that equation (87) is highly nonlinear in terms of the variable $\mu$ and, therefore, an analytical solution to $\mu$ is usually impossible except for a few simple cases. In the following, our focus is placed on a numerical solution only. For illustration, consider a three-material wedge in which materials 1 and 3 are assumed to be PZT-4, and material 2 is PZT-5 (see figure 5). The material 


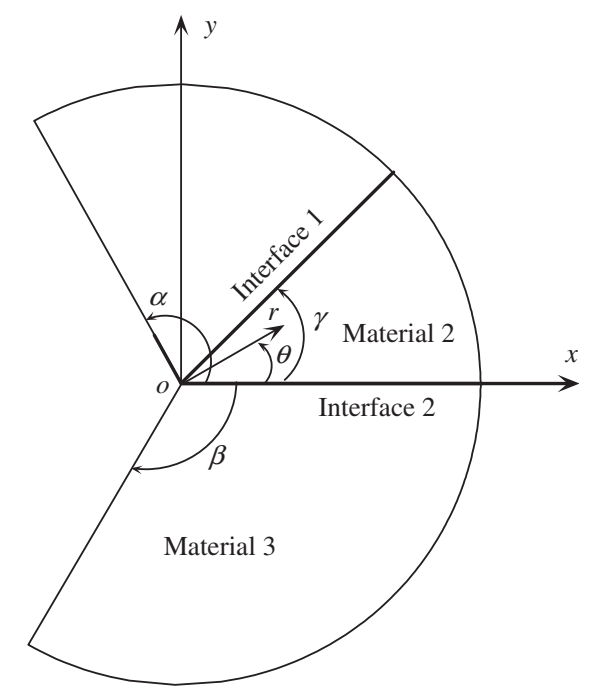

Figure 5. Piezoelectric wedge of three dissimilar materials.

Table 1. Singularity orders of a piezoelectric wedge of three dissimilar materials for different values of $\alpha_{1}$ and $e_{15}^{(1)}$ when $\alpha_{2}=\alpha_{3}=\pi / 3$ and $e^{15}=12.7 \mathrm{C} / \mathrm{N}$.

\begin{tabular}{llccll}
\hline$\alpha_{1}$ & $0.8 e_{15}^{(1)}$ & $0.9 e_{15}^{(1)}$ & $1.0 e_{15}^{(1)}$ & $1.1 e_{15}^{(1)}$ & $1.2 e_{15}^{(1)}$ \\
$\pi / 3$ & -0.269 & -0.300 & -0.324 & -0.339 & -0.339 \\
& -0.155 & -0.16 & -0.182 & -0.214 & -0.260 \\
$2 \pi / 3$ & $-0.783 \pm 0.034 \mathrm{i}$ & -0.765 & -0.812 & 0 & $-0.913 \pm 0.226 \mathrm{i}$ \\
$\pi$ & $-0.959 \pm 0.296 \mathrm{i}$ & 0 & 0 & $-0.985 \pm 0.372 \mathrm{i}$ & $-0.956 \pm 0.398 \mathrm{i}$ \\
$4 \pi / 3$ & -0.079 & -0.086 & -0.090 & -0.094 & -0.095 \\
& -0.043 & -0.047 & -0.052 & -0.06 & -0.069 \\
& $-0.967 \pm 0.355 \mathrm{i}$ & & & $-0.985 \pm 0.415 \mathrm{i}$ & $-0.963 \pm 0.435 \mathrm{i}$ \\
\hline
\end{tabular}

properties used are:

$$
\begin{aligned}
& \text { PZT-4 : } c_{44}=25.6 \times 10^{9} \mathrm{~N} / \mathrm{m}^{2}, e_{15}=12.7 \mathrm{C} / \mathrm{N}, \quad \varepsilon_{11}=6.46 \times 10^{-9} \mathrm{~F} / \mathrm{m} \\
& \text { PZT-5 }: c_{44}=21.1 \times 10^{9} \mathrm{~N} / \mathrm{m}^{2}, e_{15}=12.3 \mathrm{C} / \mathrm{N}, \quad \varepsilon_{11}=8.11 \times 10^{-9} \mathrm{~F} / \mathrm{m}
\end{aligned}
$$

As an example, table 1 lists the orders of singularity for differet values of $\alpha_{1}$ and $e_{15}^{(1)}$ when $\alpha_{2}=\alpha_{3}=\pi / 3$. It can be seen from table 1 that there exist a pair of complex or two real singularity orders for some values of $\alpha_{1}$ (e.g. two real singularity orders for $\alpha_{1}=\pi / 3$ and a pair of complex roots for $\alpha_{1}=\pi$ ) or for some values of $e_{15}^{(1)}$. It is found that, at $\alpha_{1}=(2 / 3) \pi$, the singularity order may be complex or real depending on the value of $e_{15}^{(1)}$, which indicates that $e_{15}^{(1)}$ can affect the singularity orders to some extent. In addition, the singularity order may become zero for some special values of $\alpha_{1}$ and $e_{15}^{(1)}$. This is useful when designing a multi-material wedge. It is interesting to note from table 1 that the singularity order of a three-material wedge is not equal to zero for a half-plane when $\alpha_{1}=\pi / 3$ and not equal to $-1 / 2$ for an interface crack when $\alpha_{1}=4 \pi / 3$, which is different from the results for a homogeneous 


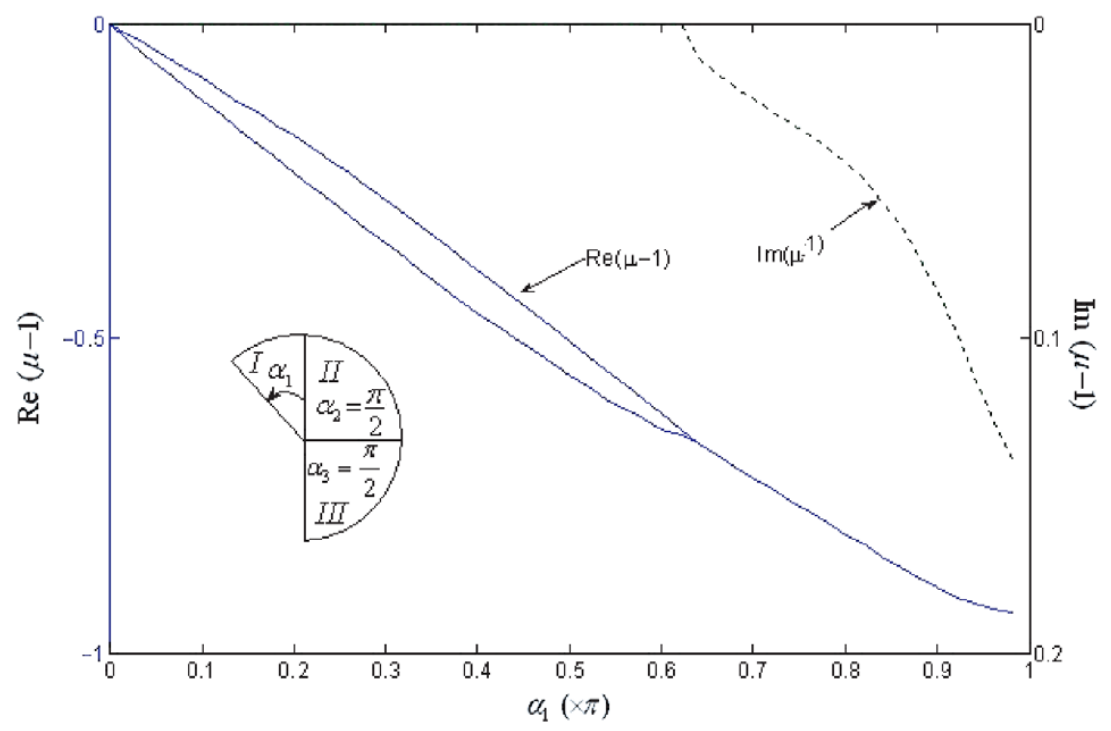

Figure 6. Variations of the singularity orders $(\mu-1)$ for a three piezoelectric materials wedge with the wedge angle $\alpha_{1}\left(\alpha_{2}=\alpha_{3}=\pi / 2, e_{15}^{(1)}=1.2 \times 12.7 \mathrm{C} / \mathrm{N}\right)$.

or bimaterial wedge. This behaviour is identical with that for an anisotropic three-material wedge [20].

It is evident from table 1 that the values of $\alpha_{1}$ and the piezoelectric constant $e_{15}^{(1)}$ have an important effect on the order of singularity. In conclusion, the order of singularity is a function of geometry and material constants for a multi-piezoelectric material wedge.

For a three piezoelectric materials wedge, the variation of singularity order with $\alpha_{1}$ has been plotted in figure 6 for $\alpha_{2}=\alpha_{3}=\pi / 2$ and in figure 7 for $\alpha_{2}=\pi / 2, \alpha_{2}=\pi$. It can be seen from figure 6 that the singularity order increases with an increase in the value of $\alpha_{1}$ and, at about $\alpha_{1}=(2 / 3) \pi$, multi-orders disappear and the complex order appears. It is observed from figure 7 that there are two singularity orders for any given value of $\alpha_{1}$, and they also increase with an increase in $\alpha_{1}$.

To prove the validity of the proposed formulation, two cases are considered as follows:

$$
\begin{array}{lll}
\text { case } 1: & \alpha_{1}=\alpha_{2}=\pi, & \alpha_{3}=0 \\
\text { case 2: } & \alpha_{1}=\alpha_{3}=\pi, & \alpha_{2}=0
\end{array}
$$

The singularity order for both cases is $-1 / 2$, which is identical with the result for an interface crack.

\subsection{Mixed multi-piezoelectric-elastic wedge}

The wedge considered in this section is obtained by replacing the piezoelectric material $i$ in figure 4 with an elastic composite, while the other materials are still assumed to be of piezoelectric material. 


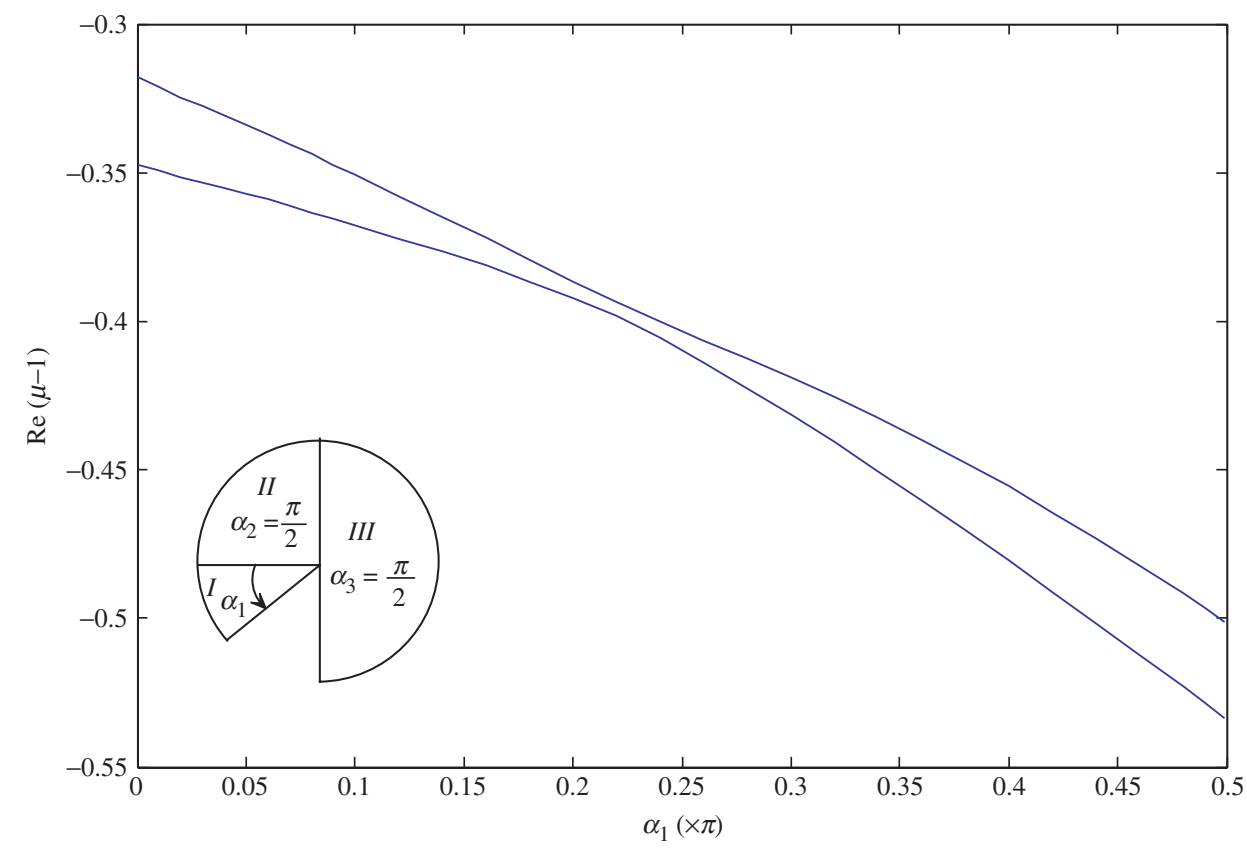

Figure 7. Variations of the singularity orders $(\mu-1)$ for a three piezoelectric materials wedge with the wedge angle $\alpha_{1}\left(\alpha_{2}=\pi / 2, \alpha_{2}=\pi, e_{15}^{(1)}=1.2 \times 12.7 \mathrm{C} / \mathrm{N}\right)$.

The boundary conditions and the continuity conditions on the bonded interface are the same as those for the multi-piezoelectric materials wedge, except that the last two equations of the continuity conditions for the interfaces between $\Omega_{i-1}$ and $\Omega_{i}, \Omega_{i}$, and $\Omega_{i+1}$ are replaced by the following equation:

$$
D_{\theta}^{(i-1)}(r, 0)=0, \quad D_{\theta}^{(i+1)}(r, 0)=0
$$

For an elastic composite wedge, the general solution can be written as:

$$
\begin{aligned}
w & =A_{1} \cos (\mu \theta)+B_{1} \sin (\mu \theta) \\
S_{r} & =\mu c_{44} A_{1} \cos (\mu \theta)+\mu c_{44} B_{1} \sin (\mu \theta) \\
S_{\theta} & =-\mu c_{44} A_{1} \sin (\mu \theta)+\mu c_{44} B_{1} \cos (\mu \theta)
\end{aligned}
$$

in which there are only two independent unknown constants $A_{1}$ and $B_{1}$, and $\mathrm{S}_{\theta}=r \tau_{\theta z}$.

From the continuity conditions (74) and (88) and the general solution (89), we can obtain the following relationships:

$$
\begin{aligned}
& A_{1(i-1)}^{(i-1)}=A_{1(i-1)}^{(i)}, \quad B_{1(i-1)}^{(i-1)}=\frac{c_{44}^{(i)}}{\Delta_{i-1}} B_{1(i-1)}^{(i)}, \quad B_{2(i-1)}^{(i-1)}=\frac{c_{44}^{(i)} e_{15}^{(i-1)}}{\Delta_{i-1} \varepsilon_{11}^{i-1}} B_{1(i-1)}^{(i)}, \\
& A_{1(i)}^{(i+1)}=A_{1(i)}^{(i)}, \quad B_{1(i)}^{(i+1)}=\frac{c_{44}^{(i)}}{\Delta_{i+1}} B_{1(i)}^{(i)}, \quad B_{2(i)}^{(i+1)}=\frac{c_{44}^{(i)} e_{15}^{(i+1)}}{\Delta_{i+1} \varepsilon_{11}^{i+1}} B_{1(i)}^{(i)}
\end{aligned}
$$


where

$$
\Delta_{i-1}=c_{44}^{(i-1)}+\frac{e_{15}^{(i-1) 2}}{\varepsilon_{11}^{(i-1)}}, \quad \Delta_{i+1}=c_{44}^{(i+1)}+\frac{e_{15}^{(i+1) 2}}{\varepsilon_{11}^{(i+1)}}
$$

Making use of equations (76) and (82), we can obtain following equations:

$$
F_{1}^{1}=\left[M_{i-1}\right] F_{i-1}^{i-1}, \quad\left\{F_{N-1}^{N}\right\}=\left[N_{i}\right]\left\{F_{i}^{i+1}\right\}
$$

where

$$
\begin{aligned}
{\left[M_{i-1}\right] } & =\left[T R_{i-1}\right]^{-1}[T(i-1, i-2)]^{-1}, \\
{\left[N_{i}\right] } & =\prod_{i=N-1}^{i}\{[R(i+1, i+2)][T(i+1, i)]\}
\end{aligned}
$$

where $\left[M_{i-1}\right]$ and $\left[N_{i}\right]$ are $4 \times 4$ matrices.

Using the coordinate transformation, the following equation can be obtained:

$$
\left\{\begin{array}{c}
A_{1(i)}^{(i)} \\
B_{1(i)}^{(i)}
\end{array}\right\}=\left[\begin{array}{cc}
\cos \left(\mu \alpha_{i}\right) & -\sin \left(\mu \alpha_{i}\right) \\
\sin \left(\mu \alpha_{i}\right) & \cos \left(\mu \alpha_{i}\right)
\end{array}\right]\left\{\begin{array}{c}
A_{1(i-1)}^{(i)} \\
B_{1(i-1)}^{(i)}
\end{array}\right\}
$$

Considering equation (94) and from the second and fourth equation of the boundary conditions (75), we can arrive at:

$$
A_{2(i-1)}^{(i-1)}=a_{1} A_{1(i-1)}^{(i)}+b_{1} B_{1(i-1)}^{(i)}, A_{2(N-1)}^{(N)}=a_{2} A_{1(i-1)}^{(i)}+b_{2} A_{2(i-1)}^{(i)}
$$

where $a_{i}$ and $b_{i}(i=1,2)$ are listed in Appendix C.

Combined use of equations (90), (92) and (95) leads to the following relationships:

$$
F_{1}^{1}=\left[M_{i-1}\right]\left[\begin{array}{cc}
1 & 0 \\
a_{1} & b_{1} \\
0 & \frac{c_{44}^{(i)}}{\Delta_{i-1}} \\
0 & \frac{c_{44}^{(i)} e_{15}^{(i-1)}}{\Delta_{i-1} \varepsilon_{11}^{(i-1)}}
\end{array}\right]\left\{\begin{array}{c}
A_{1(i-1)}^{(i)} \\
B_{1(i-1)}^{(i)}
\end{array}\right\}
$$

and

$$
F_{N-1}^{N}=\left[N_{i}\right]\left[\begin{array}{cc}
1 & 0 \\
a_{2} & b_{2} \\
0 & \frac{c_{44}^{(i)}}{\Delta_{i+1}} \\
0 & \frac{c_{44}^{(i)} e_{15}^{(i+1)}}{\Delta_{i+1} \varepsilon_{11}^{(i+1)}}
\end{array}\right]\left[\begin{array}{cc}
\cos \left(\mu \alpha_{i}\right) & -\sin \left(\mu \alpha_{i}\right) \\
\sin \left(\mu \alpha_{i}\right) & \cos \left(\mu \alpha_{i}\right)
\end{array}\right]\left\{\begin{array}{c}
A_{1(i-1)}^{(i)} \\
B_{1(i-1)}^{(i)}
\end{array}\right\}
$$


Table 2. Singularity order of piezoelectric-elastic composite wedge containing three dissimilar materials for different values of $\alpha_{2}$ and $c_{44}^{(2)}\left(\alpha_{1}=\alpha_{3}=\pi / 3\right)$.

\begin{tabular}{lcclll}
\hline$\alpha_{2}$ & $1.01 c_{44}^{(2)}$ & $2.0 c_{44}^{(2)}$ & $3.0 c_{44}^{(2)}$ & $4.0 c_{44}^{(2)}$ & $5.0 c_{44}^{(2)}$ \\
$\pi / 3$ & -0.892 & -0.892 & 0 & 0 & 0 \\
$2 \pi / 3$ & -0.946 & 0 & 0 & 0 & 0 \\
$\pi$ & 0 & 0 & 0 & 0 & 0 \\
$4 \pi / 3$ & -0.245 & -0.241 & -0.237 & -0.234 & -0.231 \\
\hline
\end{tabular}

Then, substituting equation (96) and (97) into the first and third equation of equation (75) yields:

$$
[M]\left\{\begin{array}{l}
A_{1(i-1)}^{(i)} \\
B_{1(i-1)}^{(i)}
\end{array}\right\}=0
$$

in which $[M]$ is a $2 \times 2$ matrix. The value of $\mu$ can be determined by setting the determinant of $[M]$ to be zero.

For illustration, consider a three-material wedge in which materials 1 and 3 are PZT-4 and PZT-5, respectively, and material 2 is a graphite/epoxy composite.

The properties of PZT-4 and PZT-5 can be found in section 5.1, and the material constant of the graphite/epoxy composite is $c_{44}=3.61 \times 10^{9} \mathrm{~Pa}$.

Table 2 lists the orders of singularity of the three-material wedge for different $\alpha_{2}$ and the shear modulus $c_{44}^{(2)}$. It is again found from table 2 that the singularity order is not equal to zero for the case of a half-plane and not equal to $-1 / 2$ for an interface crack. However, when $\alpha_{2}=\pi$, the singularity order vanishes. Similar to table 1 , it can also be seen that the order of singularity depends strongly on the value of $\alpha_{2}$ and the shear modulus. Knowledge of this behaviour can be applied to determine an optimum angle and optimal material constants for avoiding fracture and delamination induced by singularities of the electroelastic fields. Although only one elastic composite element is considered here for simplicity, this procedure is also suitable for the case of a wedge containing two or more elastic composite elements.

The results shown in figure 8 are for a three-material wedge with varying the wedge angle of material II (elastic material) from 0 to $\pi / 2$. It is observed that the singularity order is complex when $0 \leq \alpha_{2} \leq 0.22 \pi$ and then becomes real when $\alpha_{2}>0.22 \pi$.

\section{Conclusion}

In this paper, a symplectic model based on the Hamiltonian system has been developed for analyzing singular behaviour near the apex of a multi-dissimilar piezoelectric wedge under antiplane deformation. Explicit solutions of elastic and electric fields are obtained for the cases of composite wedge consisting of one, two and multiple piezoelectric materials and mixed piezoelectric-elastic materials. Numerical examples for cases of a three-material wedge are considered and the results show that the order of singularity of the electroelastic field near the apex of 


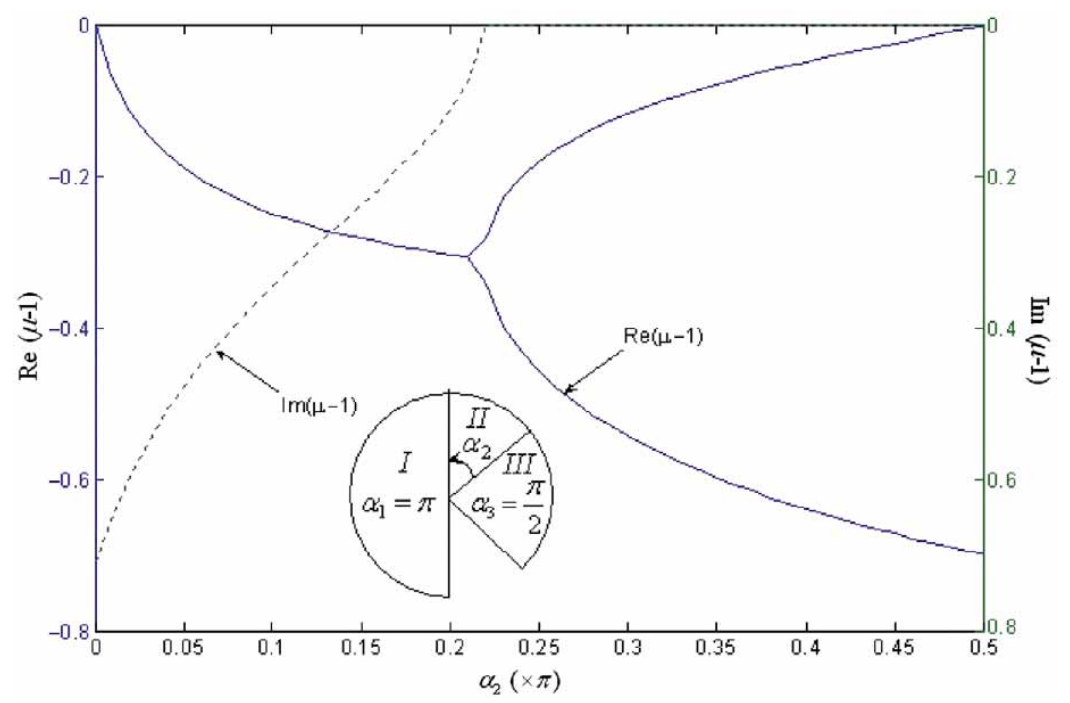

Figure 8. Variations of the singularity orders $(\mu-1)$ for a piezoelectric composite materials wedge with the wedge angle $\alpha_{2}\left(\alpha_{1}=\pi, \alpha_{3}=\pi / 2, c_{44}^{(2)}=5 \times 3.61 \times 10^{9} \mathrm{~Pa}\right)$.

the wedge is significantly affected by the geometry of the wedge, the piezoelectric constant and the shear modulus. The study also indicates that for a three-material wedge the singularity order is not equal to zero for the case of a half-plane and not equal to $-1 / 2$ for an interface crack, unlike in the case of a homogeneous wedge. In addition, this method can be easily extended to cases of in-plane deformation. This work is under way.

\section{Acknowledgements}

The authors are indebted to the two anonymous reviewers for their helpful comments and suggestions on an earlier version of this paper.

\section{Appendix A: Proof of equation (17)}

Making use of the definitions (13), (15), (16), and (18), we have

$$
\begin{aligned}
& \left\langle\boldsymbol{v}_{1}^{T}, \boldsymbol{H} \boldsymbol{v}_{2}\right\rangle=\int_{\theta_{1}}^{\theta_{2}} \boldsymbol{v}_{1}^{T} \boldsymbol{J} \boldsymbol{H} \boldsymbol{v}_{2} d \theta \\
& =\int_{\theta_{1}}^{\theta_{2}}\left\{\begin{array}{llll}
w_{1} & \varphi_{1} & S_{r 1} & S D_{r 1}
\end{array}\right\}\left[\begin{array}{cccc}
-c_{44} \frac{\partial^{2}}{\partial \theta^{2}} & -e_{15} \frac{\partial^{2}}{\partial \theta^{2}} & 0 & 0 \\
-e_{15} \frac{\partial^{2}}{\partial \theta^{2}} & \varepsilon_{11} \frac{\partial^{2}}{\partial \theta^{2}} & 0 & 0 \\
0 & 0 & -\frac{\varepsilon_{11}}{\Delta_{1}} & -\frac{e_{15}}{\Delta_{1}} \\
0 & 0 & -\frac{e_{15}}{\Delta_{1}} & \frac{c_{44}}{\Delta_{1}}
\end{array}\right]\left\{\begin{array}{c}
w_{2} \\
\varphi_{2} \\
S_{r 2} \\
S D_{r 2}
\end{array}\right\} d \theta
\end{aligned}
$$




$$
\begin{aligned}
& =\int_{\theta_{1}}^{\theta_{2}}\left\{\begin{array}{llll}
w_{1} & \varphi_{1} & S_{r 1} & S D_{r 1}
\end{array}\right\}\left[\begin{array}{c}
-c_{44} \frac{\partial^{2} w_{2}}{\partial \theta^{2}}-e_{15} \frac{\partial^{2} \varphi_{2}}{\partial \theta^{2}} \\
-e_{15} \frac{\partial^{2} w_{2}}{\partial \theta^{2}}+\varepsilon_{11} \frac{\partial^{2} \varphi_{2}}{\partial \theta^{2}} \\
-\frac{\varepsilon_{11}}{\Delta_{1}} S_{r 2}-\frac{e_{15}}{\Delta_{1}} S D_{r 2} \\
-\frac{e_{15}}{\Delta_{1}} S_{r 2}+\frac{c_{44}}{\Delta_{1}} S D_{r 2}
\end{array}\right] d \theta \\
& =\int_{\theta_{1}}^{\theta_{2}}\left\{\begin{array}{llll}
w_{2} & \varphi_{2} & S_{r 2} & S D_{r 2}
\end{array}\right\}\left[\begin{array}{c}
-c_{44} \frac{\partial^{2} w_{1}}{\partial \theta^{2}}-e_{15} \frac{\partial^{2} \varphi_{1}}{\partial \theta^{2}} \\
-e_{15} \frac{\partial^{2} w_{1}}{\partial \theta^{2}}+\varepsilon_{11} \frac{\partial^{2} \varphi_{1}}{\partial \theta^{2}} \\
-\frac{\varepsilon_{11}}{\Delta_{1}} S_{r 1}-\frac{e_{15}}{\Delta_{1}} S D_{r 1} \\
-\frac{e_{15}}{\Delta_{1}} S_{r 1}+\frac{c_{44}}{\Delta_{1}} S D_{r 1}
\end{array}\right] d \theta+C \\
& =\int_{\theta_{1}}^{\theta_{2}}\left\{\begin{array}{llll}
w_{2} & \varphi_{2} & S_{r 2} & S D_{r 2}
\end{array}\right\}\left[\begin{array}{cccc}
-c_{44} \frac{\partial^{2}}{\partial \theta^{2}} & -e_{15} \frac{\partial^{2}}{\partial \theta^{2}} & 0 & 0 \\
-e_{15} \frac{\partial^{2}}{\partial \theta^{2}} & \varepsilon_{11} \frac{\partial^{2}}{\partial \theta^{2}} & 0 & 0 \\
0 & 0 & -\frac{\varepsilon_{11}}{\Delta_{1}} & -\frac{e_{15}}{\Delta_{1}} \\
0 & 0 & -\frac{e_{15}}{\Delta_{1}} & \frac{c_{44}}{\Delta_{1}}
\end{array}\right]\left\{\begin{array}{c}
w_{1} \\
\varphi_{1} \\
S_{r 1} \\
S D_{r 1}
\end{array}\right\} d \theta+C
\end{aligned}
$$

$=\left\langle\boldsymbol{v}_{2}^{T}, \boldsymbol{H} \boldsymbol{v}_{\mathbf{1}}\right\rangle+C$

where

$$
\begin{aligned}
C= & -c_{44}\left[w_{1} \frac{\partial w_{2}}{\partial \theta}-w_{2} \frac{\partial w_{1}}{\partial \theta}\right]_{\theta_{1}}^{\theta_{2}}-e_{15}\left[\varphi_{1} \frac{\partial w_{2}}{\partial \theta}-w_{2} \frac{\partial \varphi_{1}}{\partial \theta}\right]_{\theta_{1}}^{\theta_{2}}-e_{15}\left[w_{1} \frac{\partial \varphi_{2}}{\partial \theta}-\varphi_{2} \frac{\partial w_{1}}{\partial \theta}\right]_{\theta_{1}}^{\theta_{2}} \\
& +\varepsilon_{11}\left[\varphi_{1} \frac{\partial \varphi_{2}}{\partial \theta}-\varphi_{2} \frac{\partial w_{1}}{\partial \theta}\right]_{\theta_{1}}^{\theta_{2}}
\end{aligned}
$$

$C$ is an integration constant representing the boundary condition of a wedge. In our analysis, $C$ represents the traction-charge free conditions:

$$
\tau_{\theta z}=D_{\theta}=0 \quad\left(\theta=\theta_{1}, \theta_{2}\right)
$$

If $\boldsymbol{v}_{1}, \boldsymbol{v}_{2}$ satisfy the corresponding boundary condition (A.3), then:

$$
\left\langle\boldsymbol{v}_{1}^{T}, \boldsymbol{H} \boldsymbol{v}_{2}\right\rangle=\left\langle\boldsymbol{v}_{2}^{T}, \boldsymbol{H} \boldsymbol{v}_{1}\right\rangle
$$


Therefore, according to theory of the symplectic geometry $[13,14], \boldsymbol{H}$ is the Hamiltonian matrix. It should be mentioned that equation (A.4) is valid not only for traction-charge free but also for other boundary conditions such as $\phi=0, w=0$ or the mixed boundary condition $\phi=0, \tau_{\theta z}=0$, although the derivation above is based on the homogeneous boundary condition (A.3). To this end, equation (A.2) is rewritten in the form:

$$
C=\left[w_{2} \tau_{1}+\varphi_{2} D_{1}-w_{1} \tau_{2}-\varphi_{1} D_{2}\right]_{\theta_{2}}^{\theta_{1}}
$$

in which $\tau_{i}=\left.\left(\tau_{\theta z}\right)\right|_{\theta_{i}}$ and $D_{i}=\left.\left(D_{\theta}\right)\right|_{\theta_{i}}$. It can be seen from (A.5) that equation (A.4) is valid for other boundary conditions as only two among $w, \phi, \tau, D$ equal to zero.

Appendix B: Equation for determining constants $A_{i}, B_{i}, C_{i}$, and $D_{i}(i=1-4)$

$$
\begin{aligned}
& {\left[\begin{array}{cccc}
-\mu & 0 & \frac{\varepsilon_{11}}{\Delta_{1}} & \frac{e_{15}}{\Delta_{1}} \\
0 & -\mu & \frac{e_{15}}{\Delta_{1}} & -\frac{c_{44}}{\Delta_{1}} \\
c_{44} \mu^{2} & e_{15} \mu^{2} & -\mu & 0 \\
e_{15} \mu^{2} & -\varepsilon_{11} \mu^{2} & 0 & -\mu
\end{array}\right]\left\{\begin{array}{l}
A_{1} \\
A_{2} \\
A_{3} \\
A_{4}
\end{array}\right\}=\left[\begin{array}{c}
0 \\
0 \\
2 \mu\left(c_{44} C_{1}+e_{15} C_{2}\right) \\
2 \mu\left(e_{15} C_{1}-\varepsilon_{11} C_{2}\right)
\end{array}\right]} \\
& {\left[\begin{array}{cccc}
-\mu & 0 & \frac{\varepsilon_{11}}{\Delta_{1}} & \frac{e_{15}}{\Delta_{1}} \\
0 & -\mu & \frac{e_{15}}{\Delta_{1}} & -\frac{c_{44}}{\Delta_{1}} \\
c_{44} \mu^{2} & e_{15} \mu^{2} & -\mu & 0 \\
e_{15} \mu^{2} & -\varepsilon_{11} \mu^{2} & 0 & -\mu
\end{array}\right]\left\{\begin{array}{l}
B_{1} \\
B_{2} \\
B_{3} \\
B_{4}
\end{array}\right\}=\left[\begin{array}{c}
0 \\
0 \\
-2 \mu\left(c_{44} D_{1}+e_{15} D_{2}\right) \\
-2 \mu\left(e_{15} D_{1}-\varepsilon_{11} D_{2}\right)
\end{array}\right]} \\
& {\left[\begin{array}{cccc}
-\mu & 0 & \frac{\varepsilon_{11}}{\Delta_{1}} & \frac{e_{15}}{\Delta_{1}} \\
0 & -\mu & \frac{e_{15}}{\Delta_{1}} & -\frac{c_{44}}{\Delta_{1}} \\
c_{44} \mu^{2} & e_{15} \mu^{2} & -\mu & 0 \\
e_{15} \mu^{2} & -\varepsilon_{11} \mu^{2} & 0 & -\mu
\end{array}\right]\left\{\begin{array}{l}
C_{1} \\
C_{2} \\
C_{3} \\
C_{4}
\end{array}\right\}=\left[\begin{array}{l}
0 \\
0 \\
0 \\
0
\end{array}\right]} \\
& {\left[\begin{array}{cccc}
-\mu & 0 & \frac{\varepsilon_{11}}{\Delta_{1}} & \frac{e_{15}}{\Delta_{1}} \\
0 & -\mu & \frac{e_{15}}{\Delta_{1}} & -\frac{c_{44}}{\Delta_{1}} \\
c_{44} \mu^{2} & e_{15} \mu^{2} & -\mu & 0 \\
e_{15} \mu^{2} & -\varepsilon_{11} \mu^{2} & 0 & -\mu
\end{array}\right]\left\{\begin{array}{l}
D_{1} \\
D_{2} \\
D_{3} \\
D_{4}
\end{array}\right\}=\left[\begin{array}{l}
0 \\
0 \\
0 \\
0
\end{array}\right]}
\end{aligned}
$$




\section{Appendix C: Constants $a_{i}$ and $b_{i}$}

$$
\begin{aligned}
& {\left[\begin{array}{ll}
a_{1} & b_{1}
\end{array}\right]=\frac{1}{\Delta_{m}}\left[\begin{array}{lll}
m_{1} & m_{2} & m_{3}
\end{array}\right]\left[\begin{array}{cc}
1 & 0 \\
0 & \frac{c_{44}^{(i)}}{\Delta_{i-1}} \\
& \frac{c_{44}^{(i)} e_{15}^{(i-1)}}{\Delta_{i-1} \varepsilon_{11}^{(i-1)}}
\end{array}\right]} \\
& {\left[\begin{array}{ll}
a_{2} & b_{2}
\end{array}\right]=\frac{1}{\Delta_{n}}\left[\begin{array}{lll}
n_{1} & n_{2} & n_{3}
\end{array}\right]\left[\begin{array}{cc}
1 & 0 \\
0 & \frac{c_{44}^{(i)}}{\Delta_{i+1}} \\
0 & \frac{c_{44}^{(i)} e_{15}^{(i+1)}}{\Delta_{i+1} \varepsilon_{11}^{(i+1)}}
\end{array}\right]\left[\begin{array}{cc}
\cos \left(\mu \alpha_{i}\right) & -\sin \left(\mu \alpha_{i}\right) \\
\sin \left(\mu \alpha_{i}\right) & \cos \left(\mu \alpha_{i}\right)
\end{array}\right]} \\
& m_{1}=m_{11} e_{15}^{(1)} \sin \left(\mu \alpha_{1}\right)-m_{21} \varepsilon_{11}^{(1)} \sin \left(\mu \alpha_{1}\right)-m_{31} e_{15}^{(1)} \cos \left(\mu \alpha_{1}\right)+m_{41} \varepsilon_{11}^{(1)} \cos \left(\mu \alpha_{1}\right) \\
& m_{2}=m_{13} e_{15}^{(1)} \sin \left(\mu \alpha_{1}\right)-m_{23} \varepsilon_{11}^{(1)} \sin \left(\mu \alpha_{1}\right)-m_{33} e_{15}^{(1)} \cos \left(\mu \alpha_{1}\right)+m_{43} \varepsilon_{11}^{(1)} \cos \left(\mu \alpha_{1}\right) \\
& m_{3}=m_{14} e_{15}^{(1)} \sin \left(\mu \alpha_{1}\right)-m_{24} \varepsilon_{11}^{(1)} \sin \left(\mu \alpha_{1}\right)-m_{34} e_{15}^{(1)} \cos \left(\mu \alpha_{1}\right)+m_{44} \varepsilon_{11}^{(1)} \cos \left(\mu \alpha_{1}\right) \\
& n_{1}=n_{11} e_{15}^{(1)} \sin \left(\mu \alpha_{N}\right)-n_{21} \varepsilon_{11}^{(1)} \sin \left(\mu \alpha_{N}\right)+n_{31} e_{15}^{(1)} \cos \left(\mu \alpha_{N}\right)-n_{41} \varepsilon_{11}^{(1)} \cos \left(\mu \alpha_{N}\right) \\
& n_{2}=n_{13} e_{15}^{(1)} \sin \left(\mu \alpha_{N}\right)-n_{23} \varepsilon_{11}^{(1)} \sin \left(\mu \alpha_{N}\right)+n_{33} e_{15}^{(1)} \cos \left(\mu \alpha_{N}\right)-n_{43} \varepsilon_{11}^{(1)} \cos \left(\mu \alpha_{N}\right) \\
& n_{3}=n_{14} e_{15}^{(1)} \sin \left(\mu \alpha_{N}\right)-n_{24} \varepsilon_{11}^{(1)} \sin \left(\mu \alpha_{N}\right)+n_{34} e_{15}^{(1)} \cos \left(\mu \alpha_{N}\right)-n_{44} \varepsilon_{11}^{(1)} \cos \left(\mu \alpha_{N}\right)
\end{aligned}
$$

in which $m_{i j}$ and $n_{i j}(i, j=1-4)$ are the elements of $\left[M_{i-1}\right]$ and $\left[N_{i}\right]$ respectively, and

$$
\begin{aligned}
\Delta_{m} & =\left[\varepsilon_{11}^{(1)} m_{22}-e_{15}^{(1)} m_{12}\right] \sin \left(\mu \alpha_{1}\right)+\left[\varepsilon_{11}^{(1)} m_{42}-e_{15}^{(1)} m_{32}\right] \cos \left(\mu \alpha_{1}\right) \\
\Delta_{n} & =\left[\varepsilon_{11}^{(n)} n_{22}-e_{15}^{(n)} n_{12}\right] \sin \left(\mu \alpha_{N}\right)+\left[\varepsilon_{11}^{(n)} n_{42}-e_{15}^{(n)} n_{32}\right] \cos \left(\mu \alpha_{N}\right)
\end{aligned}
$$

\section{References}

[1] Y. Matsuzaki, Smart Mater. Struct. 6 R1 (1997).

[2] Q.H. Qin, Fracture Mechanics of Piezoelectric Materials (WIT Press, Southampton, 2001).

[3] Y.L. Kang and H. Lua, Eng. Fract. Mech. 692199 (2002).

[4] Y.L. Kang and K.H. Laermann, Acta Mech. Sinica 27506 (1995).

[5] Q.H. Qin and S.W. Yu, Int. J. Solids Struct. 34581 (1997).

[6] M.L. Williams, J. Appl. Mech. 19526 (1952).

[7] C.D. Chen, Int. J. Solids Struct. 43957 (2006).

[8] X.L. Xu and R.K.N.D. Rajapakse, Int. J. Solids Struct. 373253 (2000).

[9] C.H. Chue and C.D. Chen, Int. J. Solids Struct. 393131 (2003).

[10] C.H. Chue and C.D. Chen, Arch. Appl. Mech. 72673 (2003). 
[11] C.D. Chen and C.H. Chue, Int. J. Solids Struct. 406513 (2003).

[12] H.W. Zhang and W.X. Zhong, Int. J. Solids Struct. 40493 (2003).

[13] W.X. Zhong, A New Methodology for Theory of Elasticity (Dalian University of Technology Press, Dalian, 1995), in Chinese.

[14] W.A. Yao and W.X. Zhong, Symplectic Elasticity (Higher Education Press, Beijing, 2002), in Chinese.

[15] X.S. Xu, W.X. Zhong and H.W. Zhang, Int. J. Solids Struct. 342815 (1997).

[16] W.F. Bian, F. Sun and B. Wang, Chin. J. Comput. Mech. 22411 (2005).

[17] W.A. Yao, J. Dalian Univ. Technol. 44630 (2004), in Chinese.

[18] Q.H. Qin and J.S. Wang, J. Beijing Univ. Technol. (2006), accepted for publication.

[19] Q.H. Qin, Struct. Eng. Mech. 20225 (2005).

[20] H.P. Chen, Int. J. Solids Struct. 351057 (1998). 